\title{
021713
}

\section{On non-linear Volterra integral-functional equations in several variables}

\author{
by Z. KAMONT and M. KWAPISZ (Gdañ'sk)
}

\begin{abstract}
Let $B$ be an arbitrary Banach space and $G \subset R_{+}^{n}$ be a compact set, where $R_{+}=[0,+\infty)$. Assume that the functions $F \in C\left(G \times B^{m} \times B, B\right), f_{i} \in C\left(G^{2} \times B, B\right), i=1, \ldots, m$, $\beta \in C(G, G), \alpha_{i} \in C(G, G), i=1, \ldots, m$, are given and $\beta(x) \leqslant x, \alpha_{i}(x) \leqslant x$ for $x \in G, i=1, \ldots, m$. $(C(X, Y)$ denotes the class of continuous functions defined on $X$ with range in $Y$.)

In the paper the non-linear Volterra integral-functional equation

$$
\begin{aligned}
u(x)=F\left(x, \int_{H_{1}(x)} f_{1}\left(x, s, u\left(\alpha_{1}(s)\right)\right)(d s)_{p_{1}}, \ldots\right. \\
\\
\left.\quad \ldots, \int_{H_{m}(x)} f_{m}\left(x, s, u\left(\alpha_{m}(s)\right)\right)(d s)_{p_{m}}, u(\beta(x))\right), \quad x \in G,
\end{aligned}
$$

with $H_{j}(x) \subset E(x)=\{\xi: \xi \in G, \xi \leqslant x\}$ for $x \in G, j=1, \ldots, m$, is considered.

In the first part of the paper equation (V) is discussed by means of a comparative method. If $F$ and $f_{i}$ satisfy the Lipschitz condition with respect to all variables except $x$ or $x, s$, respectively, then, under certain additional assumptions concerning the functions $\beta, \alpha_{j}$ and the Lipschitz coefficients, it is proved that there exists exactly one (in a certain class of functions) continuous solution of (V). This solution is the limit of the sequence of successive approximations. It is not assumed that the Lipschitz coefficient $k$ of the function $F$ with respect to the last variable satisfies the condition $k<1$.

The second part of the paper deals with equation (V) considered in a finite dimensional Banach space. A theorem on the existence of at least one solution of equation (V) is proved. Also in this case conditions milder than $k<1$ are assumed.
\end{abstract}

Introduction. Let $B$ be an arbitrary Banach space with norm $\|\cdot\|$. Denote by $C(X, Y)$ the set of all continuous functions defined in $X$ taking values in $Y, X, Y$ being arbitrary metric spaces. For $x=\left(x_{1}, \ldots, x_{n}\right)$, $y=\left(y_{1}, \ldots, y_{n}\right) \in R^{n}\left(R^{n}\right.$ - real Euclidean space of dimension $\left.n\right)$ we define $x \leqslant y$ as $x_{i} \leqslant y_{i}$ for $i=1, \ldots, n$. We denote by $|\cdot|$ the Euclidean norm in $R^{n}$. Let $G \subset R_{+}^{n}$ be a compact set, where $R_{+}=[0,+\infty)$. Let

$$
E(x)=\{\xi: \xi \in G, \xi \leqslant x\} \text {. }
$$

Assume that the functions $F \in C\left(G \times B^{m} \times B, B\right), f_{i} \in C\left(G^{2} \times B, B\right), i=1, \ldots, m$, $\beta \in C(G, G), \alpha_{i} \in C(G, G), i=1, \ldots$ are given and $\beta(x) \leqslant x, \alpha_{i}(x) \leqslant x$ for $x \in G, i=1, \ldots, m$.

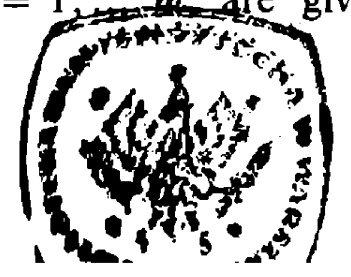


We shall consider the non-linear Volterra integral-functional equation

$$
\begin{aligned}
u(x)=F\left(x, \int_{H_{1}(x)} f_{1}\left(x, s, u\left(\alpha_{1}(s)\right)\right)(d s)_{p_{1}}, \ldots\right. \\
\left.\ldots, \int_{H_{m}(x)} f_{m}\left(x, s, u\left(\alpha_{m}(s)\right)\right)(d s)_{p_{m}}, u(\beta(x))\right), \quad x \dot{x} G,
\end{aligned}
$$

where $H_{j}(x) \subset E(x)$ for $x \in G, j=1, \ldots, m$.

We assume further that $H_{j}(x)$ is contained in a $p_{j}$-dimensional hyperplane $\left(1 \leqslant p_{j} \leqslant n\right)$, parallel to the coordinate axes, and it is Lebesgue measurable, considered as a $p_{j}$-dimensional set. Let $L_{p_{j}}\left(H_{j}(x)\right)$ denotes the $p_{j}$-dimensional Lebesgue measure of $H_{j}(x)$. We assume that $p_{j}$ does not depend on $x$.

If a $p_{j}$-dimensional hyperplane containing the set $H_{j}(x)$ and being parallel to the coordinate axes is defined by the equations

$$
x_{t_{1}}=\dot{x}_{t_{1}}, \quad x_{t_{2}}=\dot{x}_{t_{2}}, \quad x_{t_{r}}=\dot{x}_{t_{r}}, \quad r=n-p_{j},
$$

then $\int_{H_{j}(x)} g(x, s)(d s)_{p_{j}}$, where $\dot{s}=\left(s_{1}, \ldots, s_{n}\right)$, denotes the $p_{j}$-dimensional Lebesgue integral in the space $O x_{m_{1}} x_{m_{2}} \ldots x_{m_{p j}}, m_{i} \in\left\{\{1, \ldots, n\}-\left\{t_{1}, \ldots, t_{r}\right\}\right\}$, and $s_{t_{1}}=\dot{x}_{t_{1}}, s_{t_{2}}=\dot{x}_{i_{2}}, \ldots, s_{t_{r}}=\dot{x}_{t_{r}}$.

Let $A^{\prime}=\left\{i: p_{i}=n\right\}, B^{\prime}=\left\{i: 1 \leqslant p_{i}<n\right\}$. By changing notation, if necessary, we may assume that $A^{\prime}=\left\{1, \ldots, k_{0}\right\}, B^{\prime}=\left\{k_{0}+1, \ldots, m\right\}$.

We define the sets $\sigma_{j} \subset\{1, \ldots, n\}, j=1, \ldots, m$, in the following way: if the axis $O x_{i}$ is parallel to the $p_{j}$-dimensional hyperplane in which the set $H_{j}(x)$ is contained, then $i \in \sigma_{j}$. Put $\bar{\sigma}_{j}=\{1, \ldots, n\}-\sigma_{j}$.

For each $x \in G$ and $j=1, \ldots, m$ we introduce the set $G_{j}(x)$ by

$$
\begin{aligned}
& G_{j}(x)=\left\{s: s=\left(s_{1}, \ldots, s_{n}\right), s_{t_{i}}=\dot{x}_{t_{i}}\right. \\
& \text { for } t_{i} \in \sigma_{j}, \\
& \left.0 \leqslant s_{t_{i}} \leqslant \varphi_{t_{i}}^{(j)}(x) \text { for } t_{i} \in \bar{\sigma}_{j}\right\},
\end{aligned}
$$

where $\left(\varphi_{t_{1}}^{(j)}, \ldots, \varphi_{t_{p}}^{(j)}\right)=\varphi_{j} \in C\left(G, R_{+}^{p_{j}}\right), t_{i} \in \bar{\sigma}_{j}$, and $H_{j}(x) \subset G_{j}(x) \subset E(x)$. The $p_{j}$-dimensional Lebesgue-measure of $G_{j}(x)$ satisfies $L_{p_{j}}\left(G_{j}(x)\right)=\prod_{s \in \sigma_{j}} \varphi_{s}^{(j)}(x)$.

We adopt the following notations:

$$
\begin{gathered}
\int_{H(x)} f(x, s, z(\alpha(s))) d s=\left(\int_{H_{1}(x)} f_{1}\left(x, s, z\left(\alpha_{1}(s)\right)\right)(d s)_{p_{1}}, \ldots\right. \\
\left.\ldots, \int_{H_{m}(x)} f_{m}\left(x, s, z\left(\alpha_{m}(s)\right)\right)(d s)_{p_{m}}\right) \\
L(G(x))=\left(L_{p_{1}}\left(G_{1}(x)\right), \ldots, L_{p_{m}}\left(G_{m}(x)\right)\right)
\end{gathered}
$$

if $K=\left(K_{1}, \ldots, K_{m}\right) \in C\left(G, R^{m}\right)$, then

$$
K(x) \int_{H(x)} f(x, s, z(\alpha(s))) d s=\sum_{j=1}^{m} K_{j}(x) \int_{H_{j}(x)} f_{j}\left(x, s, z\left(\alpha_{j}(s)\right)\right)(d s)_{p_{j}}, \quad x \in G,
$$


and

$$
K(x) \int_{H(x)} z(\alpha(s)) d s=\sum_{j=1}^{m} K_{j}(x) \int_{H(x)} z\left(\alpha_{j}(s)\right)(d s)_{p_{j}}, \quad x \in G .
$$

For $K \in C\left(G, R^{m}\right)$ we define

$$
K(x) L(G(x))=\sum_{j=1}^{m} K_{j}(x) L_{p_{j}}\left(G_{j}(x)\right), \quad x \in G .
$$

Equation (1) will be written briefly

$$
u(x)=F\left(x, \int_{H(x)} f(x, s, u(\alpha(s))) d s, u(\beta(x))\right), \quad x \in G .
$$

There are various problems which lead to Volterra integral-functional equations of type (2). Perhaps the simplest problem in the theory of differential equations which leads to such an equation with $n=1$ is the initial-value problem for the ordinary differential-functional equation of the neutral type

$$
u^{\prime}(t)=F\left(t, u\left(\alpha_{1}(t)\right), \ldots, u\left(\alpha_{m}(t)\right), u^{\prime}(\beta(t))\right), \quad t \in[0, a], u(0)=u_{0} .
$$

Therefore equation (2) is a generalization of equations which have been considered in paper [3] and also of some cases of equations considered in [1], [2], [5], [7], [15].

The various initial value problems for the partial hyperbolic differentialfunctional equation of the neutral type

$$
\begin{array}{r}
z_{x y}(x, y)=F\left(x, y, z\left(\alpha_{1}^{(0)}(x, y), \alpha_{2}^{(0)}(x, y)\right), z_{x}\left(\alpha_{1}^{(1)}(x, y), \alpha_{2}^{(1)}(x, y)\right),\right. \\
\left.z_{y}\left(\alpha_{1}^{(2)}(x, y), \alpha_{2}^{(2)}(x, y)\right), z_{x y}\left(\beta_{1}(x, y), \beta_{2}(x, y)\right)\right)
\end{array}
$$

can be reformulated in terms of Volterra integral-functional equations. Let us consider as an example the Darboux problem, where the domain is a rectangle $\{(x, y): x \in[0, a], y \in[0, b]\}$, and where initial values $u(x, 0)$ $=\sigma(x), x \in[0, a], u(0, y)=\tau(\dot{y}), y \in[0, b]$ are prescribed. The Volterra integral-functional equation corresponding to that problem is

$$
\begin{array}{r}
u(x, y)=F\left(x, y, \sigma\left(\alpha_{1}^{(0)}(x, y)\right)+\tau\left(\alpha_{2}^{(0)}(x, y)\right)-\sigma(0)+\int_{H_{0}(x, y)} u(s, t) d s d t,\right. \\
\sigma^{\prime}\left(\alpha_{1}^{(1)}(x, y)\right)+\int_{H_{1}(x, y)} u(s, t) d t, \tau^{\prime}\left(\alpha_{2}^{(2)}(x, y)\right)+\int_{H_{2}(x, y)} u(s, t) d s, \\
\left.u\left(\beta_{1}(x, y), \beta_{2}(x, y)\right)\right), \quad(x, y) \in[0, a] \times[0 ; b],
\end{array}
$$

where

$$
\begin{aligned}
& H_{0}(x, y)=\left\{(s, t): s \in\left[0, \alpha_{1}^{(0)}(x, y)\right], t \in\left[0, \alpha_{2}^{(0)}(x, y)\right]\right\} \\
& H_{1}(x, y)=\left\{(s, t): s=\alpha_{1}^{(1)}(x, y), t \in\left[0, \alpha_{2}^{(1)}(x, y)\right]\right\} \\
& H_{2}(x, y)=\left\{(s, t): s \in\left[0, \alpha_{1}^{(2)}(x, y)\right], t=\alpha_{2}^{(2)}(x, y)\right\}
\end{aligned}
$$


Therefore our equation is a generalization of the equation which was considered in paper [6] and of an adequate case of the equation disscused in [4].

The Cauchy problem and the Goursat problem for hyperbolic differentialfunctional equations leads to a Volterra integral-functional equation of type (2) (see [13]).

Similar initial value problems for equations in more than two variables and problems for equations of higher order can be reformulated in terms of Volterra integral-functional'equations.

As a particular case of equation (2) we can obtain the system of Volterra integral equations which was considered by $W$. Walter in paper [12] and monograph [13]. These papers contain the extensive bibliography concerning Volterra integral equations.

In the case when $u$ is a function of several variables equation (2) is a generalization of equations which have been considered in [8]-[11].

In this paper we give theorems concerning the existence and uniqueness of continuous solutions of (2) in a certain class of functions.

The paper is divided into two parts. In the first part we investigate equation (2) by means of the comparative method. A general formulation of this method can be found in paper [14]. If we assume that $F$ and $f_{i}$ satisfy the Lipschitz condition with respect to all variables except $x$ or $x, s$, respectively, then we prove, under certain additional assumptions concerning the functions $\beta, \alpha_{j}$ and the Lipschitz coefficients, that there exists exactly one (in a certain class of functions) continuous solution of (2). This solution is the limit of a sequence of successive approximations. This result is obtained by means of the comparative method.

The essential fact in our considerations is that we do not assume that the Lipschitz coefficient $k$ of the function $F$ with respect to the last variable satisfies the condition $k<1$ (see Lemmas 4-9). If $k<1$, then we have a theorem on the existence and uniqueness of solutions of (2), which can be obtained by means of the Banach fixed-point theorem.

The second part of the paper concerns equation (2) considered in a finite dimensional Banach space. We prove here a theorem on the existence of at least one solution of equation (2). In this case it is an important fact that we also do not assume that the Lipschitz coefficient $k$ of the function $F$ with respect to the last variable satisfies the condition $k<1$ (see Lemma 14). This part of the paper is an extension of the result contained in paper [3], where an equation of type (2) with the unknown function of one variable was considered.

Remark 1. Let

$$
G^{*}(x)=\{\xi: \xi \leqslant x\}, \quad \tilde{G}=\bigcup_{s \in G} G^{*}(s) .
$$


(We do not assume that $G^{*}(x) \subset G$.) Suppose that the functions $F \in C\left(G \times B^{m} \times B, B\right), f_{i} \in C\left(G^{2} \times B, B\right), \alpha_{i} \in C(\widetilde{G}, \widetilde{G}), i=1, \ldots, m, \beta \in C(G, \widetilde{G})$, $\varphi \in C(\tilde{G}-G, B)$ are given and $\beta(x) \leqslant x, \alpha_{i}(x) \leqslant x$ for $x \in G, i=1, \ldots, m$.

Let us consider the equation

$$
\begin{aligned}
u(x) & =F\left(x, \int_{H_{1}(x)} f_{1}\left(x, s, u\left(\alpha_{1}(s)\right)\right)(d s)_{p_{1}}, \ldots\right. \\
& \left.\ldots, \int_{H_{m}(x)} f_{m}\left(x, s, u\left(\alpha_{m}(s)\right)\right)(d s)_{p_{m}}, u(\beta(x))\right), \quad x \in G, \\
u(x) & =\varphi(x) \quad \text { for } x \in \tilde{G}-G,
\end{aligned}
$$

where $H_{j}(x) \subset \bar{G}$. (We do not assume that the sets $H_{j}(x)$ satisfy the condi tion $H_{j}(x) \subset G$.)

We want to point out that equation $\left(1^{\prime}\right)$ is equivalent to some equation of type (1). We shall prove this only for the case $m=1$, i.e., for the equation

$$
\begin{aligned}
& u(x)=F\left(x, \int_{H(x)} f(x, s, u(\alpha(s)))(d s)_{p}, u(\beta(x))\right), \quad x \in G, \\
& u(x)=\varphi(x) \quad \text { for } x \in \bar{G}-G .
\end{aligned}
$$

We define for $x \in G$

$$
\tilde{H}(x)=\{s: s \in H(x) \cap G \text { and } \alpha(s) \in G\}, \quad \tilde{H}(x)=H(x)-\tilde{H}(x) .
$$

Then we have

$$
\int_{H(x)} f(x, s, u(\alpha(s)))(d s)_{p}=\int_{\tilde{H}(\dot{x})} f(x, s, u(\alpha(s)))(d s)_{p}+\int_{\tilde{\tilde{H}}(x)} f(x, s, \varphi(\alpha(s)))(d s)_{p} .
$$

Lèt

$$
\tilde{\Delta}=\{x \in G: \beta(x) \in G\}, \quad \tilde{\tilde{\Delta}}=\{x \in G: \beta(x) \in \bar{G}-G\} .
$$

Let $\bar{\beta}$ be a function satisfying the following conditions:

(a) $\tilde{\beta} \in C(G, G)$,

(b) $\tilde{\beta}(x)=\beta(x)$ for $x \in \tilde{\Delta}, \tilde{\beta}(x) \leqslant x$ for $x \in G$.

Put

$$
\tilde{F}(x, u, v)= \begin{cases}F(x, u, v) & \text { for } x \in \tilde{\Delta} \\ F(x, u, \varphi(\beta(x))) & \text { for } x \in \tilde{\tilde{\Delta}}\end{cases}
$$

Now equation $\left(1^{\prime \prime}\right)$ is equivalent to the equation

$$
\begin{array}{r}
u(x)=\tilde{F}\left(x, \int_{\tilde{H}(x)} f(x, s, u(\alpha(s)))(d s)_{p}+\int_{\tilde{\hat{H}}(x)} f(x, s, \varphi(\alpha(s)))(d s)_{p}, u(\tilde{\beta}(x))\right), \\
x \in G,
\end{array}
$$

which is of type (1). 


\section{PART I}

1. Assumptions. Let $x \in G, h \in R^{n}, x+h \in G, i \in B^{\prime}$. Suppose that the set $H_{i}(x)$ is contained in a $p_{i}$-dimensional hyperplane $\left(1 \leqslant p_{i}<n\right)$ parallel to the $n-p_{i}$ coordinate axes. We denote this hyperplane by $S_{i}(x)$. Let the set $H_{i}(x+h)$ be contained in a $p_{i}$-dimensional hyperplane $S_{i}(x+h)$ parallel to the hyperplane $S_{i}(x)$. There exists a vector $t_{i}(x, h) \in R^{n}$ such that the set $-t_{i}(x, h)+H_{i}(x+h)$ is contained in $S_{i}(x)$.

We introduce

Assumption $H_{1}$ (see [12], p. 970; [13], p. 134). Suppose that:

$1^{\circ}$ for $i \in A^{\prime}$ we have $\lim _{h \rightarrow 0} L_{n}\left[H_{i}(x)-H_{i}(x+h)\right]=0$ uniformly with respect to $x \in G$ (the sign $\dot{-}$ denotes the symmetric difference of two sets),

$2^{\circ}$ for $i \in B^{\prime}$ we have, uniformly with respect to $x \in G$,

(a) $\lim _{h \rightarrow 0} t_{i}(x, h)=\cdot 0$,

(b) $\lim _{h \rightarrow 0} L_{p_{i}}\left[H_{i}(x) \div\left(-t_{i}(x, h)+H_{i}(x+h)\right)\right]=0$.

Remark 2. If $x \in S_{i}(x)$ for $x \in G$ and $i \in B^{\prime}$, we may assume that $t_{i}(x, h)=h$. Condition (a) of Assumption $\mathrm{H}_{1}$ is satisfied in this case.

Assumption $\mathrm{H}_{2}$. Suppose that:

$1^{\circ}$ the functions $k, \tilde{h} \in C\left(G, R_{+}\right), K=\left(K_{1}, \ldots, K_{m}\right) \in C\left(G, R_{+}^{m}\right), \beta \in C(G, G)$ are given and $\beta(x) \leqslant x$ for $x \in G$,

$2^{\circ}$ we have

$$
\tilde{m}(x)=\sum_{i=0}^{\infty} k^{(i)}(x) \tilde{h}\left(\beta^{(i)}(x)\right)<+\infty \quad \text { for } x \in G
$$

where

$k^{(0)}(x)=1 \quad$ for $x \in G, \quad k^{(i+1)}(x)=k(x) k^{(i)}(\beta(x)) \quad$ for $x \in G, i=0,1,2, \ldots$, $\beta^{(0)}(x)=x \quad$ for $x \in G, \quad \beta^{(i+1)}(x)=\beta\left(\beta^{(i)}(x)\right) \quad$ for $x \in G, i=0,1,2, \ldots$,

$3^{\circ}$ we have

$$
M(x)=\sum_{i=0}^{\infty} k^{(i)}(x)\left[K\left(\beta^{(i)}(x)\right) L\left(G\left(\beta^{(i)}(x)\right)\right)\right]<+\infty \quad \text { for } x \in G,
$$

$4^{\circ} M, \tilde{m} \in C\left(G, R_{+}\right)$, the function

$$
\tilde{M}(x)=\sum_{i=0}^{\infty} k^{(i)}(x)\left[\sum_{j=1}^{m} K_{j}\left(\beta^{(i)}(x)\right) L_{p_{j}}\left(G_{j}\left(\beta^{(i)}(x)\right)\right)\left(\prod_{s \in \tilde{\sigma}_{j}} x_{s}\right)^{-1}\right]
$$

is bounded for $x \in G$. 
We adopt the following notation:

$$
\begin{aligned}
\bar{m}(x) & =\sum_{i=0}^{\infty} k^{(i)}(x) \bar{h}\left(\beta^{(i)}(x)\right), \\
(V z)(x) & =\sum_{i=0}^{\infty} k^{(i)}(x)\left[K\left(\beta^{(i)}(x)\right) \int_{H\left(\beta^{(i)}(x)\right)} z(\alpha(s)) d s\right] .
\end{aligned}
$$

Remark 3. If

(a) conditions $1^{\circ} \mathbf{H}_{2}-3^{\circ} \mathbf{H}_{2}$ are satisfied,

(b) $\bar{h} \in C\left(G, R_{+}\right), \bar{h}(x) \leqslant \bar{h}(x)$ for $x \in G$,

(c) $z$ is a non-negative and upper-semicontinuous function,

then $\bar{m}$ and $V z$ are functions defined in $G$.

\section{The main lemma.}

Lemma 1. If Assumptions $\mathrm{H}_{1}, \mathrm{H}_{2}$ are satisfied and $\bar{h} \in C\left(G, R_{+}\right)$, $\bar{h}(x) \leqslant \bar{h}(x)$ for $x \in G$, then:

$1^{\circ}$ There exist solutions $\bar{z}, \tilde{z} \in C\left(G, R_{+}\right)$of the equations

$$
z(x)=\bar{m}(x)+(V z)(x), \quad x \in G
$$

and

$$
z(x)=\tilde{m}(x)+(V z)(x), \quad x \in G,
$$

respectively. The solutions $\bar{z}$ and $\tilde{z}$ of (5) and (6), respectively, are unique in the set $M\left(G, R_{+}\right)$of non-negative upper-semicontinuous functions.

$2^{\circ}$ The functions $\bar{z}$ and $\tilde{z}$ are solutions of the equations

$$
z(x)=K(x) \int_{H(x)} z(\alpha(s)) d s+k(x) z(\beta(x))+\bar{h}(x), \quad x \in G
$$

and

$$
z(x)=K(x) \int_{H(x)} z(\alpha(s)) d s+k(x) z(\beta(x))+\dot{\bar{h}}(x), \quad x \in G,
$$

respectively. Moreover, these solutions are unique in the class $\tilde{M}\left(G, R_{+}, \tilde{z}\right)$, where

$$
\tilde{M}\left(G, R_{+}, \tilde{z}\right)=\left\{z: z \in M\left(G, R_{+}\right) \text {and inf }[c: z(x) \leqslant c \tilde{z}(x)]<+\infty\right\} .
$$

The function $\tilde{z}$ satisfies the condition

$$
\lim _{r \rightarrow \infty} k^{(r)}(x) \tilde{z}\left(\beta^{(r)}(x)\right)=0 \quad \text { uniformly with respect to } x \in G .
$$

$3^{\circ}$ The function $z(x)=0$ for $x \in G$ is the unique solution of the inequality

$$
z(x) \leqslant K(x) \int_{H(x)} z(\alpha(s)) d s+k(x) z(\beta(x)), \quad x \in G,
$$

in the class $\bar{M}\left(G, R_{+}, \bar{z}\right)$. 
$4^{\circ}$ If $k, \bar{h}, \bar{h}, K, \beta$ are non-decreasing in $G$ and $H_{j}(x) \subset H_{j}(\bar{x})$ for $x<\bar{x}$, $x, \bar{x} \in G, j=1,2, \ldots, m$, then $\bar{z}$ and $\bar{z}$ are non-decreasing in $G$.

Proof. We shall show that equation (5) has exactly one solution in the set $M\left(G, R_{+}\right)$. Let $T$ be the operator defined by the right-hand side of equation (5). We prove that $T: M\left(G, R_{+}\right) \rightarrow M\left(G, R_{+}\right)$. Let $z \in M\left(G, R_{+}\right)$, $v_{i j}(x)=\int_{H_{j}\left(\beta^{(i)}(x)\right)} z\left(\alpha_{j}(s)\right)(d s)_{p_{j}}$. Then there exists a sequence $\left\{z_{r}\right\}$ such that $z_{r} \in C\left(G, R_{+}\right)$and

$$
\begin{array}{r}
z_{r+1}(x) \leqslant z_{r}(x), \quad x \in G, r=1,2, \ldots, \\
\text { and } \quad z(x)=\lim _{r \rightarrow \infty} z_{r}(x), \quad x \in G .
\end{array}
$$

Let $v_{i j}^{(r)}(x)=\int_{H_{j}\left(\beta^{(i)}(x)\right)} z_{r}\left(\alpha_{j}(s)\right)(d s)_{p j}, \quad x \in G, \quad i=1, \ldots, n, \quad j=1, \ldots, m$, $r=1,2, \ldots$ The functions $v_{i j}^{(r)}$ are continuous in $G$ (cf. [12], p. 972), and $v_{i j}^{(r+1)}(x) \leqslant v_{i j}^{(r)}(x)$. From (11) and by the Lebesgue theorem on integration of non-increasing sequences we have $v_{i j}(x)=\lim _{r \rightarrow \infty} v_{i j}^{(r)}(x), i=1, \ldots, n$, $j=1, \ldots, m, x \in G$. Since $v_{i j}$ is the limit of the non-increasing sequence of continuous functions, we see that $v_{i j} \in M\left(G, R_{+}\right)$. It follows from Dini's theorem and from assumptions $2^{\circ}$ of $\mathrm{H}_{1}, 3^{\circ}$ of $\mathrm{H}_{1}$ that series (3) and (4) are uniformly convergent in $G$. From this fact and by the conditions

$$
\begin{aligned}
& k^{(i)}(x) \bar{h}\left(\beta^{(i)}(x)\right) \leqslant k^{(i)}(x) \tilde{h}\left(\beta^{(i)}(x)\right), \quad i=0,1,2, \ldots, x \in G, \\
& k^{(i)}(x)\left[K\left(\beta^{(i)}(x)\right) \int_{H\left(\beta^{(i)}(x)\right)} z(\alpha(s)) d s\right] \\
& \leqslant\left[\sup _{x \in G} z(x)\right] k^{(i)}(x)\left[K\left(\beta^{(i)}(x)\right) \cdot L\left(G\left(\beta^{(i)}(x)\right)\right)\right], \quad i=0,1,2, \ldots, x \in G,
\end{aligned}
$$

we infer the uniform convergence in $G$ of the following series:

$$
\sum_{i=0}^{\infty} k^{(i)}(x) \bar{h}\left(\beta^{(i)}(x)\right), \quad \sum_{i=0}^{\infty} k^{(i)}(x)\left[K\left(\beta^{(i)}(x)\right) \int_{H\left(\beta^{(i)}(x)\right)} z(\alpha(s)) d s\right] .
$$

Hence we get $\bar{m} \in C\left(G, R_{+}\right), V z \in M\left(G, R_{+}\right)$and consequently, $T: M\left(G, R_{+}\right)$ $\rightarrow M\left(G, R_{+}\right)$.

Now we prove that the operator $T$ is a contraction. Let

$$
\|z\|_{0}=\max _{x \in G}\left[e^{-\lambda\left(x_{1}+\ldots+x_{n}\right)}|z(x)|\right]
$$

where $z \in M\left(G, R_{+}\right)$, and $\lambda>\Lambda=\max \left[1, \sup _{x \in G} \tilde{M}(x)\right]$. For $z, w \in M\left(G, R_{+}\right)$ 
we get

$$
\begin{aligned}
|(T z)(x)-(T w)(x)| & \leqslant \sum_{i=0}^{\infty} k^{(i)}(x)\left[K\left(\beta^{(i)}(x)\right) \int_{H\left(\beta^{(i)}(x)\right)}|z(\alpha(s))-w(\alpha(s))| d s\right] \\
& \leqslant\|z-w\|_{0} \sum_{i=0}^{\infty} k^{(i)}(x)\left[K\left(\beta^{(i)}(x)\right) \int_{H\left(\beta^{(i)}(x)\right)} e^{j\left(s_{1}+\ldots+s_{n}\right)} d s\right] .
\end{aligned}
$$

We have the following estimates:

$$
\begin{aligned}
\int_{H_{j}\left(\beta^{(i)}(x)\right)} e^{\lambda\left(s_{1}+\ldots+s_{n}\right)}(d s)_{p_{j}} & \leqslant \exp \left(\lambda \sum_{p \in \sigma_{j}} x_{p}\right) \int_{G_{j}\left(\beta^{(i)}(x)\right)} \exp \left(\lambda \sum_{p \in \bar{\sigma}_{j}} s_{p}\right)(d s)_{p_{j}} \\
& =\exp \left(\lambda \sum_{p \in \sigma_{j}} x_{p}\right) \prod_{p \in \sigma_{j}}\left\{\frac{1}{\lambda}\left(\exp \left[\lambda \varphi_{p}^{(j)}\left(\beta^{(i)}(x)\right)\right]-1\right)\right\} \\
& \leqslant \frac{1}{\lambda} \exp \left(\lambda \sum_{p \in \sigma_{j}} x_{p}\right) \prod_{p \in \bar{\sigma}_{j}}\left\{\exp \left[\lambda x_{p} \frac{\varphi_{p}^{(j)}\left(\beta^{(i)}(x)\right)}{x_{p}}\right]-1\right\} \\
& \leqslant \frac{1}{\lambda} e^{\lambda\left(x_{1}+\ldots+x_{n}\right)} L_{p_{j}}\left(G_{j}\left(\beta^{(i)}(x)\right)\right)\left(\prod_{p \in \sigma_{j}} x_{p}\right)^{-1} .
\end{aligned}
$$

The last inequality is a consequence of the obvious inequality

$$
e^{x}-1 \leqslant \gamma e^{t} \quad \text { for } \gamma \in[0,1], t \geqslant 0 .
$$

Finally, we obtain

$$
\begin{aligned}
\mid(T z)(x) & -(T w)(x) \mid \\
\leqslant & \frac{1}{\lambda}\|z-\dot{w}\|_{0} \sum_{i=0}^{\infty} k^{(i)}(x)\left[\sum_{j=1}^{m} K_{j}\left(\beta^{(i)}(x)\right) L_{p_{j}}\left(G_{j}\left(\beta^{(i)}(x)\right)\right) \times\right. \\
& \left.\times\left(\prod_{p \in \bar{\sigma}_{j}} x_{p}\right)^{-1}\right] e^{j\left(x_{1}+\ldots+x_{n}\right)} \\
\leqslant & \frac{1}{\lambda} \Lambda\|z-w\|_{0} e^{i\left(x_{1}+\ldots+x_{n}\right)},
\end{aligned}
$$

and consequently

$$
\|T z-T w\|_{0} \leqslant \frac{\Lambda}{\lambda}\|z-w\|_{0}
$$

Since $A<\lambda$, then by the Banach fixed point theorem we infer that equation (5) has a unique solution $\bar{z}$ being an upper-semicontinuous function.

We prove that $\bar{z} \in C\left(G, R_{+}\right)$. The solution $\bar{z}$ of equation (5) is the limit of the sequence $\left\{z_{r}\right\}$ which is defined in the following way:

$$
\begin{gathered}
z_{0} \in M\left(G, R_{+}\right), \quad z_{0}-\text { arbitrarily fixed, } \\
z_{r+1}(x)=\bar{m}(x)+\left(V z_{r}\right)(x), \quad x \in G, r=0,1,2, \ldots
\end{gathered}
$$


For $z \in M\left(G, R_{+}\right)$we define $\left(V^{0} z\right)(x)=z(x),\left(V^{i+1} z\right)(x)=\left(V\left(V^{i} z\right)\right)(x), x \in G$, $i=0,1, \ldots$ We easily see that

$$
z_{r+1}(x)=\sum_{i=0}^{r}\left(V^{i} \bar{m}\right)(x)+\left(V^{r+1} z_{0}\right)(x) .
$$

$\left\{V^{r} z_{0}\right\}$ is the sequence of successive approximations for the equation $z(x)=(V z)(x)$. Since this equation has a solution $z(x)=0, x \in G$, which is unique in the set $M\left(G, R_{+}\right)$, it follows that

$$
\lim _{r \rightarrow \infty}\left(V^{r} z_{0}\right)(x)=0 \quad \text { uniformly with respect to } x \in G \text {. }
$$

Since the functions $V^{i} \bar{m}$ are continuous in $G$ and the sequence $\left\{z_{r}\right\}$ is uniformly convergent, it follows from (12) that

$$
\bar{z}(x)=\sum_{i=0}^{\infty}\left(V^{i} \bar{m}\right)(x)
$$

is a continuous function in $G$. This completes the proof of assertion $1^{\circ}$ of Lemma 1.

Now we shall prove assertion $2^{\circ}$. At first we prove that equality (9) holds true. It is easy to check that functions $k^{(r)}$ and $\beta^{(r)}$ satisfy the conditions

$$
k^{(r)}(x) k^{(i)}\left(\beta^{(r)}(x)\right)=k^{(r+i)}(x), \quad \beta^{(i)}\left(\beta^{(r)}(x)\right)=\beta^{(r+i)}(x)
$$

Formulas (13) and (6) imply

$$
x \in G, r, i=0,1, \ldots
$$

$$
\begin{aligned}
k^{(r)}(x) \tilde{z}\left(\beta^{(r)}(x)\right)=\sum_{i=0}^{\infty} k^{(r+i)}(x) \tilde{h}\left(\beta^{(r+i)}(x)\right)+ \\
\quad+\sum_{i=0}^{\infty} k^{(r+i)}(x)\left[K\left(\beta^{(r+i)}(x)\right) \int_{H(\beta(r+i)(x))} \tilde{z}(\alpha(s)) d s\right] .
\end{aligned}
$$

This last equality and (3), (4) imply (9): The uniform convergence of $\left\{k^{(r)}(x) \tilde{z}\left(\beta^{(r)}(x)\right)\right\}$ follows from the uniform convergence of series (3) and (4).

We observe that any solution of equation (5) is a solution of (7). Indeed, if $\bar{z}$ is a solution of equation (5), we have

$$
\begin{aligned}
& \bar{z}(x)-K(x) \int_{H(x)} \bar{z}(\alpha(s)) d s-k(x) \bar{z}(\beta(x)) \\
&=\sum_{i=0}^{\infty} k^{(i)}(x) \bar{h}\left(\beta^{(i)}(x)\right)+\sum_{i=0}^{\infty} k^{(i)}(x)\left[K\left(\beta^{(i)}(x)\right) \int_{H\left(\beta^{(i)}(x)\right)} \bar{z}(\alpha(s)) d s\right]- \\
&-K(x) \int_{H(x)} \bar{z}(\alpha(s)) d s-k(x)\left\{\sum_{i=0}^{\infty} k^{(i)}(\beta(x)) \bar{h}\left(\beta^{(i)}(\beta(x))\right)+\right. \\
&\left.+\sum_{i=0}^{\infty} k^{(i)}(\beta(x))\left[K\left(\beta^{(i)}(\beta(x))\right) \int_{H\left(\beta\left(s^{(i)}(\beta(x))\right)\right.} \bar{z}(\alpha(s)) d s\right]\right\} \equiv \bar{h}(x),
\end{aligned}
$$

which means that $\bar{z}$ is a solution of (7). 
Now we prove that $\bar{z}$ is a unique solution of (7) in the set $\tilde{M}\left(G, R_{+}, \bar{z}\right)$. In fact, if $\bar{z} \in \tilde{M}\left(G, R_{+}, \tilde{z}\right)$ is a solution of (7), then for $r=1,2, \ldots$ and any $x \in G$ the equality

$$
\begin{aligned}
& \bar{z}(x)=\sum_{i=0}^{r-1} k^{(i)}(x)\left[K\left(\beta^{(i)}(x)\right) \int_{H(\beta(i)(x))} \overline{\bar{z}}(\alpha(s)) d s\right]+ \\
& +\sum_{i=0}^{r-1} k^{(i)}(x) \bar{h}\left(\beta^{(i)}(x)\right)+k^{(r)}(x)^{\bar{z}}\left(\beta^{(r)}(x)\right)
\end{aligned}
$$

holds.

Since $\overline{\bar{z}} \in \bar{M}\left(G, R_{+}, \tilde{z}\right)$, we have for some $c \in R_{+}: 0 \leqslant \overline{\bar{z}}(x) \leqslant c \tilde{z}(x)$ for $x \in G$. Now, according to (9), we obtain

$$
\lim _{r \rightarrow \infty} k^{(r)}(x) \bar{z}\left(\beta^{(r)}(x)\right)=0 \quad \text { uniformly with respect to } x \in G \text {. }
$$

If we let $r \rightarrow \infty$ in relation (14), we obtain

$$
\overline{\bar{z}}(x)=\bar{m}(x)+(V \bar{z})(x), \quad x \in G,
$$

i.e. $\overline{\bar{z}}$ is the solution of equation (5). This equation has only the solution $\bar{z}$; hence it results that $\bar{z}=\bar{z}$. Thus the prool of $2^{\circ}$ is completed.

Now we are going to prove $3^{\circ}$. Let us suppose that $z^{*} \in \bar{M}\left(G, R_{+}, \tilde{z}\right)$ and $z^{*}$ is a solution of inequality (10). We obtain easily for $r=1,2, \ldots$ and $x \in G$

$$
z^{*}(x) \leqslant \sum_{i=0}^{r-1} k^{(i)}(x)\left[K\left(\beta^{(i)}(x)\right) \int_{H\left(\beta^{(i)}(x)\right)} z^{*}(\alpha(s)) d s\right]+k^{(r)}(x) z^{*}\left(\beta^{(r)}(x)\right) .
$$

Since $z^{*} \in \tilde{M}\left(G, R_{+}, \tilde{z}\right)$, we have for some $c \in R_{+}: 0 \leqslant z^{*}(x) \leqslant c \tilde{z}(x)$ for $x \in G$. By (9) the last inequalities implies

$$
\lim _{r \rightarrow \infty} k^{(r)}(x) z^{*}\left(\beta^{(r)}(x)\right)=0 \quad \text { uniformly with respect to } x \in G .
$$

Letting $r$ in (16) tend to $\infty$ we get

$$
z^{*}(x) \leqslant\left(V z^{*}\right)(x), \quad x \in G .
$$

Let $\left\{z_{r}\right\}$ be the sequence defined in the following way:

(18) $z_{0}(x)=\tilde{z}(x), \quad x \in G, \quad z_{r+1}(x)=\left(V z_{r}\right)(x), \quad x \in G, r=0,1,2, \ldots$

From assertions $1^{\circ}$ and $2^{\circ}$ of this Lemma and from (18) it follows that

$$
0 \leqslant z_{r+1}(x) \leqslant z_{r}(x), \quad x \in G, r=0,1,2, \ldots
$$

and

$$
\lim _{r \rightarrow \infty} z_{r}(x)=0 \quad \text { uniformly in } G .
$$

Further, by (17), we obtain

$$
z^{*}(x) \leqslant c z_{r}(x), \quad x \in G, r=0,1, \ldots
$$


The last formula together with (20) gives $z^{*}(x)=0$ for $x \in G$, which completes the proof of assertion $3^{\circ}$.

The simple proof of assertion $4^{\circ}$ is omitted.

LEMMA 2. If Assumptions $\mathrm{H}_{1}$ and $\mathrm{H}_{2}$ are satisfied and the sequence $\left\{w_{r}\right\}$ is defined by the formulas

$$
\begin{array}{r}
w_{0}(x)=\tilde{z}(x), \quad w_{r+1}(x)=K(x) \int_{H(x)} w_{r}(\alpha(s)) d s+k(x) w_{r}(\beta(x)), \\
x \in G, r=0,1, \ldots
\end{array}
$$

then

$$
\begin{gathered}
0 \leqslant w_{r+1}(x) \leqslant w_{r}(x) \leqslant w_{0}(x), \quad x \in G, r=0,1 ; 2, \ldots, \\
\lim _{r \rightarrow \infty} w_{r}(x)=0 \quad \text { uniformly with respect to } x \in G
\end{gathered}
$$

Proof. Relations (22) follow by induction. The convergence of the sequence $\left\{w_{r}\right\}$ is implied by $(22)$. Since $w_{r} \in C\left(G, R_{+}\right)$, it follows that the limit $\bar{w}$ of the sequence $\left\{w_{r}\right\}$ is an upper-semicontinuous function. From (21) it follows that $\bar{w}$ satisfies inequality (10). According to assertion $3^{\circ}$ of Lemma 1 we have $\bar{w}(x)=0$ for $x \in G$. The uniform convergence of the sequence $\left\{w_{r}\right\}$ follows from Dini's theorem.

3. The existence and uniqueness of solutions of equation (2). We introduce the following

Assumption $\mathrm{H}_{3}$. Suppose that:

$1^{o}$ There exist functions $l_{i} \in C\left(G, R_{+}\right), i=1, \ldots, m, k \in C\left(G, R_{+}\right)$such that

$$
\|F(x, u, v)-F(x, \bar{u}, \bar{v})\| \leqslant \sum_{i=1}^{m} l_{i}(x)\left\|u_{i}-\bar{u}_{i}\right\|+k(x)\|v-\bar{v}\|,
$$

where $u=\left(u_{1}, \ldots, u_{m}\right), \bar{u}=\left(\bar{u}_{1}, \ldots, \bar{u}_{m}\right), x \in G, u, \bar{u} \in B^{m}, v, \bar{v} \in B$.

$2^{\circ}$ There exist functions $\bar{l}_{i} \in C\left(G, R_{+}\right), i=1, \ldots, m$, such that

$$
\left\|f_{i}(x, s, z)-f_{i}(x, s, \bar{z})\right\| \leqslant \bar{l}_{i}(x)\|z-\bar{z}\|, \quad x, s \in G, z, \bar{z} \in B .
$$

$3^{0}$ There exists a function $u_{0} \in C(G, B)$ such that Assumption $\mathrm{H}_{2}$ is fulfilled for $\tilde{h}, K$ defined by relations

$$
\begin{gathered}
\tilde{h}(x)=\left\|F\left(x, \int_{H(x)} f\left(x, s, u_{0}(\alpha(s))\right) d s, u_{0}(\beta(x))\right)-u_{0}(x)\right\|, \\
K(x)=\left(l_{1}(x) \bar{l}_{1}(x), \ldots, l_{m}(x) \bar{l}_{m}(x)\right),
\end{gathered}
$$

and for $k$ defined by condition' $1^{\circ}$ of Assumption $\mathrm{H}_{3}$. 
Lemma 3. If Assumptions $\mathrm{H}_{1}$ and $\mathrm{H}_{3}$ are satisfied and the sequence $\left\{u_{r}\right\}$ is defined by the relations

$$
u_{r+1}(x)=F\left(x, \int_{H(x)} f\left(x, s, u_{r}(\alpha(s))\right) d s, u_{r}(\beta(x))\right), \quad x \in G, r=0,1,2, \ldots,
$$

where $u_{0}$ is given by condition $3^{\circ}$ of Assumption $\mathrm{H}_{3}$, then

$$
\begin{aligned}
\left\|u_{r}(x)-u_{0}(x)\right\| & \leqslant \tilde{z}(x), \quad x \in G, r=0,1,2, \ldots, \\
\left\|u_{r+p}(x)-u_{r}(x)\right\| & \leqslant w_{r}(x), \quad x \in G, r=0,1,2, \ldots,
\end{aligned}
$$

where $\tilde{z}$ is defined in Lemma 1 , and the sequence $\left\{w_{r}\right\}$ is defined by relations (21).

Proof. We prove that $(25)$ is fulfilled. For $r=0$ this inequality is evidently satisfied. If we assume that $\left\|u_{r}(x)-u_{0}(x)\right\| \leqslant \tilde{z}(x)$ for $x \in G$, then

$$
\begin{aligned}
\left\|u_{r+1}(x)-u_{0}(x)\right\| \leqslant & \| F\left(x, \int_{H(x)} f\left(x, s, u_{r}(\alpha(s))\right) d s, u_{r}(\beta(x))\right)- \\
& \quad-F\left(x, \int_{H(x)} f\left(x, s, u_{0}(\alpha(s))\right) d s, u_{0}(\beta(x))\right)+\tilde{h}(x) \\
\leqslant & K(x) \int_{H(x)} \tilde{z}(\alpha(s)) d s+k(x) \tilde{z}(\beta(x))+\tilde{h}(x)=\tilde{z}(x), \quad x \in G .
\end{aligned}
$$

Now we obtain (25) by induction.

Next we prove (26). From (21) and (25) it follows that (26) is satisfied for $r=0, p=0,1,2, \ldots, x \in G$. If we assume that (26) holds for arbitrarily fixed $r$ and any $p=0,1,2, \ldots, x \in G$, then

$$
\begin{aligned}
&\left\|u_{r+1+p}(x)-u_{r+1}(x)\right\| \leqslant \| F\left(x, \int_{H(x)} f\left(x, s, u_{r+p}(\alpha(s))\right) d s, u_{r+p}(\beta(x))\right)- \\
&-\left.F\left(x, \int_{H(x)} f\left(x, s, u_{r}(\alpha(s))\right) d s, u_{r}(\beta(x))\right)\right|_{i} \\
& \leqslant K(x) \int_{H(x)} w_{r}(\alpha(s)) d s+k(x) w_{r}(\beta(x))=w_{r+1}(x) .
\end{aligned}
$$

Now (26) follows by induction with respect to $r$.

We have the following

THEOREM 1. If Assumptions $\mathrm{H}_{1}$ and $\mathrm{H}_{3}$ are satisfied, then there exists a solution $\bar{u} \in C(G, B)$ of equation (2) such that

$$
\begin{array}{ll}
\left\|\bar{u}(x)-u_{0}(x)\right\| \leqslant \bar{z}(x), & x \in G, \\
\left\|\bar{u}(x)-u_{r}(x)\right\| \leqslant w_{r}(x), & x \in G, r=0,1,2, \ldots,
\end{array}
$$

where $u_{r}$ and $w_{r}$, are defined by formulas (24) and (21), respectively. The solution $\bar{u}$ of (2) is unique in the class

$$
X(G, B) \stackrel{d \rho}{=} \bigcup_{c \geq 0}\left\{u: u \in C(G, B),\left\|u(x)-u_{0}(x)\right\| \leqslant c \tilde{z}(x), x \in G\right\}
$$


Proof. It follows from (23) and (26) that the sequence $\left\{u_{r}\right\}$ is uniformly convergent in $G$ to a certain function $\bar{u} \in C(G, B)$. Obviously $\bar{u}$ is a solution of (2). The estimates (27) and (28) are implied by (25) and (26), respectively. To prove that the solution $\bar{u}$ of (2) is unique in the class considered, let us suppose that there exists another solution $\bar{u}$ of equation (2) and $\bar{u} \in X(G, B)$. It is easy to check that the function $z(x)=\|\bar{u}(x)-\bar{u}(x)\|$ is an element of the set $\tilde{M}\left(G, R_{+}, \tilde{z}\right)$ and

$$
z(x) \leqslant K(x) \int_{H(x)} z(\alpha(s)) d s+k(x) z(\beta(x)), \quad x \in G .
$$

By assertion $3^{\circ}$ of Lemma 1 we get $z(x)=0$ for $x \in G$, and hence $\bar{u}(x)=\bar{u}(x)$ for $x \in G$. Thus the proof of Theorem 1 is complete.

4. Continuous dependence of solutions on the right-hand side of equation (2). Let us consider another equation:

$$
v(x)=\tilde{F}\left(x, \int_{\tilde{H}(x)} f(x, s, v(\tilde{\alpha}(s))) d s, v(\tilde{\beta}(x))\right), \quad x \in G,
$$

where the functions $\tilde{F}, \tilde{f}=\left(\tilde{f}_{1}, \ldots, \tilde{f}_{m}\right), \tilde{\alpha}=\left(\tilde{\alpha}_{1}, \ldots, \tilde{\alpha}_{m}\right), \tilde{\beta}$ and the sets $\tilde{H}(x)$ $=\left(\tilde{H}_{1}(x), \ldots, \tilde{H}_{m}(x)\right)$ have the same properties as $F, f, \alpha, \beta, H(x)$, which are formulated Assumptions $\mathrm{H}_{1}, \mathrm{H}_{3}$. Suppose that $\bar{u}$ and $\bar{v}$ are solutions of equations (2) and (29), respectively. Let $\tilde{r} \in C\left(G, R_{+}\right)$be a function such that $\|\bar{u}(x)-\bar{v}(x)\| \leqslant \tilde{r}(x)$ for $x \in G$. Let

$$
\begin{gathered}
q(x) \stackrel{\text { del }}{=} \| F\left(x, \int_{H(x)} f(x, s, \bar{v}(\alpha(s))) d s, \bar{v}(\beta(x))\right)- \\
-\tilde{F}\left(x, \int_{\tilde{H}(x)} f(x, s, \bar{v}(\bar{\alpha}(s))) d s, \bar{v}(\tilde{\beta}(x))\right) \|, \\
\tilde{h}_{1}(x)=\max [\tilde{r}(x), q(x), \tilde{h}(x)], \quad x \in G .
\end{gathered}
$$

Now we have the following

THeOrem 2. If the functions $F, f, \alpha, \beta$ and $\tilde{F}, \tilde{f}, \tilde{\alpha}, \tilde{\beta}$ and $\tilde{h}_{1}$ satisfy Assumption $\mathrm{H}_{3}$ and the sets $H(x), \tilde{H}(x), x \in G$, satisfy Assumption $\mathrm{H}_{1}$, then there exists a solution $\bar{w} \in C\left(G, R_{+}\right)$of the equation

$$
z(x)=K(x) \int_{H(x)} z(\alpha(s)) d s+k(x) z(\beta(x))+q(x), \quad x \in G,
$$

such that

$$
\|\bar{u}(x)-\bar{v}(x)\| \leqslant \bar{w}(x) \quad \text { for } \dot{x} \in G .
$$

Proof. Let $\bar{z}$ be a solution of the equation

$$
z(x)=K(x) \int_{H(x)}^{\prime} z(\alpha(s)) d s+k(x) z(\beta(x))+\tilde{h}_{1}(x), \quad x \in G .
$$


Put

$$
\begin{gathered}
w_{0}(x)=\bar{z}(x), \quad x \in G, \\
w_{r+1}(x)=K(x) \int_{H(x)} w_{r}(\alpha(s)) d s+k(x) w_{r}(\beta(x))+q(x), \quad x \in G, r=0,1,2, \ldots
\end{gathered}
$$

By induction we get $0 \leqslant w_{r+1}(x) \leqslant w_{r}(x) \leqslant \bar{z}(x), x \in G, r=0,1,2, \ldots$ From these inequalities we see that the sequence $\left\{w_{r}\right\}$ is convergent to the solution $\bar{w}$ of equation (30) and $0 \leqslant \bar{w}(x) \leqslant \bar{z}(x)$ for $x \in G$. However, in view of Lemma 1 , there exists only one solution of this equation in the class of upper-semicontinuous functions satisfying the condition $0 \leqslant \bar{w}(x)$ $\leqslant \bar{z}(x)$ for $x \in G$.

Now, we show that

$$
\|\bar{u}(x)-\bar{v}(x)\| \leqslant w_{r}(x) \quad \text { for } x \in G, r=0,1,2, \ldots
$$

Since $\tilde{r}(x) \leqslant \tilde{h}_{1}(x) \leqslant \bar{z}(x)=w_{0}(x), x \in G$, it follows that (32) is satisfied for $r=0$ and $x \in G$. If we assume that $\|\bar{u}(x)-\bar{v}(x)\| \leqslant w_{r}(x)$ for $x \in G$ and for some $r$, then

$$
\begin{aligned}
&\|\bar{u}(x)-\bar{v}(x)\| \leqslant \| F\left(x, \int_{H(x)} f(x, s, \bar{u}(\alpha(s))) d s, \bar{u}(\beta(x))\right)- \\
&-F\left(x, \int_{H(x)} f(x, s, \bar{v}(\alpha(s))) d s, \bar{v}(\beta(x))\right) \|+ \\
&+\| F\left(x, \int_{H(x)} f(x, s, \bar{v}(\alpha(s))) d s, \bar{v}(\beta(x))\right)- \\
&-\tilde{F}\left(x, \int_{\bar{H}(x)} f(x, s, \bar{v}(\tilde{\alpha}(s))) d s, \bar{v}(\bar{\beta}(x))\right) \| \\
& \leqslant K(x) \int_{H(x)}\|\bar{u}(\alpha(s))-\bar{v}(\alpha(s))\| d s+k(x)\|\bar{u}(\beta(x))-\bar{v}(\beta(x))\|+q(x) \quad x \in G .
\end{aligned}
$$

Now (32) follows by induction. Letting $r \rightarrow \infty$ in (32), we get estimation (31).

5. Some effective conditions. We give here simple sufficient conditions for assumptions $2^{\circ}-4^{\circ}$ in $\mathrm{H}_{2}$ to be satisfied.

Lemma 4. Assume that

$1^{\circ} k(x) \leqslant \bar{k}, K(x)=\left(K_{1}(x), \ldots, K_{m}(x)\right) \leqslant\left(\bar{K}_{1}, \ldots, \bar{K}_{m}\right), \bar{k}, \bar{K}_{i} \in R_{+}$,

$2^{\circ} \varphi_{i}(x)=\left(\varphi_{t_{1}}^{(i)}(x), \ldots, \varphi_{t_{p_{i}}}^{(i)}(x)\right) \leqslant\left(\bar{\alpha}_{t_{1}}^{(i)} x_{i_{1}}, \ldots, \bar{\alpha}_{i_{p_{i}}}^{(i)} x_{t_{p_{i}}}\right)$, where $\bar{\alpha}_{i_{j}}^{(i)} \in R_{+}$, $t_{j} \in \sigma_{i}, \vec{\alpha}_{t_{j}}^{(i)} \leqslant 1$,

$3^{\circ} \beta(x)=\left(\beta_{1}(x), \ldots, \beta_{n}(x)\right) \leqslant\left(\bar{\beta}_{1} x_{1}, \ldots, \bar{\beta}_{n} x_{n}\right), \bar{\beta}_{i} \in R_{+}, \bar{\beta}_{i} \leqslant 1$,

$4^{\mathrm{o}} \sum_{i=0}^{\infty} \bar{k}^{i} \tilde{h}\left(\bar{\beta}^{i} x_{1}, \ldots, \bar{\beta}^{i} x_{n}\right)<+\infty$,

$5^{\circ} k \prod_{s \in \sigma_{j}} \bar{\beta}_{s}<1$ for $j=1,2, \ldots, m$. 
Under these assumptions conditions $2^{\circ}-4^{\circ}$ of Assumption $\mathrm{H}_{2}$ are satisfied.

Proof. By induction we easily obtain the estimates $k^{(i)}(x) \leqslant \bar{k}^{i}, x \in G$, $i=0,1,2, \ldots$ and $\beta^{(i)}(x) \leqslant\left(\bar{\beta}_{1}^{i} x_{1}, \ldots, \bar{\beta}_{n}^{i} x_{n}\right), x \in G, i=0,1,2, \ldots$ From these inequalities we get the following estimation for series (3):

$$
\sum_{i=0}^{\infty} k^{(i)}(x) \tilde{h}\left(\beta^{(i)}(x)\right) \leqslant \sum_{i=0}^{\infty} \bar{k}^{i} \tilde{h}\left(\bar{\beta}_{1}^{i} x_{1}, \ldots, \bar{\beta}_{n}^{i} x_{n}\right), \quad x \in G
$$

Now $4^{\circ}$ implies condition $2^{\circ}$ of Assumption $\mathrm{H}_{2}$. Since

$$
\begin{aligned}
& \sum_{i=0}^{\infty} k^{(i)}(x)\left[K\left(\beta^{(i)}(x)\right) L\left(G\left(\beta^{(i)}(x)\right)\right)\right] \\
&=\sum_{j=1}^{m}\left[\sum_{i=0}^{\infty} k^{(i)}(x) K_{j}\left(\beta^{(i)}(x)\right) L_{p_{j}}\left(G\left(\beta^{(i)}(x)\right)\right)\right],
\end{aligned}
$$

then for a fixed index $j$ we have

$$
\begin{aligned}
& \sum_{i=0}^{\infty} k^{(i)}(x) K_{j}\left(\beta^{(i)}(x)\right) L_{p_{j}}\left(G\left(\beta^{(i)}(x)\right)\right) \leqslant \sum_{i=0}^{\infty} \bar{k}^{i} \bar{K}_{j} \prod_{s \in \sigma_{j}} \varphi_{s}^{(j)}\left(\beta^{(i)}(x)\right) \\
& \leqslant \bar{K}_{j} \sum_{i=0}^{\infty} \bar{k}^{i} \prod_{s \in \bar{\sigma}_{j}} \bar{\alpha}_{s}^{j} \cdot \bar{\beta}_{s}^{(i)} \cdot x_{s} \leqslant \bar{K}_{j}\left(\prod_{s \in \bar{\sigma}_{j}} \bar{\alpha}_{s}^{j}\right)\left(\prod_{s \in \sigma_{j}} x_{s}\right) \sum_{i=0}^{\infty}\left(\bar{k} \prod_{s \in \bar{\sigma}_{j}} \bar{\beta}_{s}\right)^{i} .
\end{aligned}
$$

Hence and from assumption $5^{\circ}$ of this Lemma it follows that condition $3^{\circ}$ of Assumption $\mathrm{H}_{2}$ is satisfied. From Dini's theorem and from the last inequalities it follows that condition $4^{\circ}$ of Assumption $\mathrm{H}_{2}$ is satisfied, too.

Remark 4. Suppose that conditions $1^{\circ}-4^{\circ}$ of Lemma 4 are satisfied. A sufficient condition for the existence of a solution of equations of the type (2) given in Lemma 11 in [11] is of the form

$$
\bar{k} \max _{1 \leqslant s \leqslant n} \bar{\beta}_{s}<1 \text {. }
$$

We see easily that condition $5^{\circ}$ of Lemma 4 is more general than condition (33).

By a similar argument we can prove the following lemmas:

\section{LEMMA 5. If}

$1^{\circ} k(x) \leqslant \bar{k}, K_{j}(x) \leqslant \bar{K}_{1} x_{1}+\ldots+\bar{K}_{n} x_{n}, j=1,2, \ldots, m, \vec{k}, \bar{K}_{i} \in R_{+}$,

$2^{\circ}$ assumptions $2^{\circ}-4^{\circ}$ of Lemma 4 are satisfied, then conditions $2^{\circ}-4^{\circ}$ of Assumption $\mathrm{H}_{2}$ are fulfilled.

LEMMA 6. If

$1^{\circ} G=[0, a], a=\left(a_{1}, \ldots, a_{n}\right), a_{i}>0, i=1, \ldots, n$,

$2^{\circ} k(x) \leqslant \bar{k}_{1} x_{1}+\ldots+\bar{k}_{n} x_{n}, \quad K(x)=\left(K_{1}(x), \ldots, K_{n}(x)\right) \leqslant\left(\bar{K}_{1}, \ldots, \bar{K}_{n}\right)$, $\bar{k}_{i}, \bar{K}_{i} \in R_{+}$,

$3^{\prime \prime}$ assumptions $2^{\circ}, 3^{\circ}$ of Lemma 4 are satisfied, 
$4^{\circ} \bar{k}_{1} \bar{\beta}_{1} a_{1}+\ldots+\bar{k}_{n} \bar{\beta}_{n} a_{n}<1$,

then conditions $2^{\circ}-4^{\circ}$ of Assumption $\mathbf{H}_{2}$ are fulfilled.

LEMMA 7. If

$1^{\circ} k(x) \leqslant \bar{k}, K_{j}(x) \leqslant \bar{K}_{1} x_{1}+\ldots+\bar{K}_{n} x_{n}, j=1, \ldots, m, \bar{k}, \bar{K}_{i} \in R_{+}$,

$2^{\circ} \quad \varphi_{j}(x) \leqslant\left(\bar{\alpha}_{i_{1}}^{(j)} x_{t_{1}}^{2}, \ldots, \bar{\alpha}_{i_{p_{j}}}^{(j)} x_{i_{p j}}^{2}\right), t_{i} \in \bar{\sigma}_{j}, \bar{\alpha}_{t_{s}}^{(j)} \in R_{+}$,

$3^{\circ}$ assumptions $3^{\circ}, 4^{\circ}$ of Lemma 4 are satisfied,

$4^{\circ} \bar{k} \bar{\beta}_{i}\left(\prod_{s \in \bar{\sigma}_{j}} \bar{\beta}_{s}\right)^{2}<1, i=1,2, \ldots, n, j=1,2, \ldots, m$,

then conditions $2^{\circ}-4^{\circ}$ of Assumption $\mathrm{H}_{2}$ are satisfied.

LEMMA 8. If

$1^{\circ} G=[0, a], \quad a=\left(a_{1}, \ldots, a_{n}\right), \quad 0<a_{i} \leqslant 1, \quad i=1, \ldots, n, \prod_{s \in \bar{\sigma}_{j}} a_{s}^{2}<1$, $j=1, \ldots, m$,

$2^{\circ} \beta(x) \leqslant\left(x_{1}^{2}, \ldots, x_{n}^{2}\right)$,

$3^{\circ}$ assumptions $1^{\circ}, 2^{\circ}$ of Lemma 4 are satisfied,

$4^{\circ} \sum_{i=0}^{\infty} \bar{k}^{i} \tilde{h}\left(x_{1}^{2^{i}}, \ldots, x_{n}^{2^{i}}\right)<\infty$,

then conditions $2^{\circ}-4^{\circ}$ of Assumption $\mathrm{H}_{2}$ are fulfilled.

LEMMA 9. If

$1^{\circ}$ assumptions $1^{\circ}, 2^{\circ}, 3^{\circ}$ of Lemma 4 are satisfied,

$2^{o} \tilde{h}(x) \leqslant h x^{p}, h=$ const, $x^{p}=x_{1}^{p} \cdot x_{2}^{p} \cdot \ldots \cdot x_{n}^{p}$,

$3^{0} \bar{k}\left(\prod_{s \in \bar{\sigma}_{j}} \bar{\beta}_{s}\right)^{v}<1$, where $v=\min [1, p]$,

then conditions $2^{\circ}-4^{\circ}$ of Assumption $\mathrm{H}_{2}$ are fulfilled.

\section{PART II}

In this part of the paper we give sufficient conditions for the existence of at least one continuous solution of equation (2) considered in a finite dimensional Banach space $B$. Now we do not assume the Lipschitz condition for the function $F$ with respect to $u_{i}$ for $i \in A^{\prime}$ 'and for the functions $f_{i}$ with respect to $z$ for $i \in A^{\prime}$ (see Assumption $\mathrm{H}_{3}$ in Part I).

1. Assumptions. We introduce the following

Assumption $\mathrm{H}_{4}$. Suppose that:

$1^{\circ}$ There exist functions $h_{i} \in C\left(G, R_{+}\right), i=0,1, \ldots, m, g, \bar{h}_{i}, \bar{g}_{i} \in C\left(G, R_{+}\right)$, $i=1,2, \ldots, m$, such that

$$
\|F(x, u, v)\| \leqslant \sum_{i=1}^{m} h_{i}(x)\left\|u_{i}\right\|+h_{0}(x)\|v\|+g(x), \quad x \in G,
$$

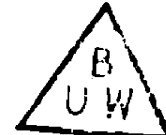


where $u=\left(u_{1}, \ldots, u_{m}\right) \in B^{m}, v \in B, B$ is a finite dimensional Banach space and $\left\|f_{i}(x, s, z)\right\| \leqslant \bar{h}_{i}(x)\|z\|+g_{i}(x), \quad i=1,2, \ldots, m, x, s \in G$.

$2^{\circ}$ There exist functions $l_{i} \in C\left(G, R_{+}\right), i=k_{0}+1, \ldots, m, k \in C\left(G, R_{+}\right)$and $\bar{l}_{i} \in C\left(G, R_{+}\right), i=k_{0}+1, \ldots, m$, such that

$$
\left\|F\left(x, u_{A}, u_{B}, v\right)-F\left(x, u_{A}, \bar{u}_{B}, \bar{v}\right)\right\| \leqslant \sum_{i=k_{0}+1}^{m} l_{i}(x)\left\|u_{i}-\bar{u}_{i}\right\|+k(x)\|v-\bar{v}\|,
$$

where $x \in G, u_{A}=\left(u_{1}, \ldots, u_{k_{0}}\right), u_{B}=\left(u_{k_{0}+1}, \ldots, u_{m}\right), u_{i}, \bar{u}_{i}, v, \bar{v} \in B$, and

$$
\begin{aligned}
\left\|f_{i}(x, s, z)-f_{i}(x, s, \bar{z})\right\| \leqslant \bar{l}_{i}(x)\|z-\bar{z}\| \quad \text { for } i=k_{0}+1, \ldots, m, \\
x, s \in G, z, \bar{z} \in B .
\end{aligned}
$$

$3^{\circ}$ Assumption $\mathrm{H}_{2}$ is fulfilled for $\tilde{h}, K$ defined by the relations

$$
\begin{aligned}
K(x) & =\left(h_{1}(x) \bar{h}_{1}(x), \ldots, h_{m}(x) \bar{h}_{m}(x)\right), \\
\tilde{h}(x) & =\sum_{i=1}^{m}\left[h_{i}(x) \bar{g}_{i}(x) \sup _{x \in(i} L_{p_{i}}\left(H_{i}(x)\right)\right]+g(x)
\end{aligned}
$$

and for $k$ defined in condition $2^{\circ}$ of Assumption $\mathbf{H}_{4}$.

Assumption $H_{5}$. We assume that the functions $D_{j}, d_{i}, \bar{d}_{i} ; \bar{d}_{i}, d_{i}, \bar{\omega}_{s}$, $\bar{\omega}_{s} \in C\left(R_{+}, R_{+}\right), i=1, \ldots, m, j=0,1, \ldots, m+1, s=k_{0}+1, \ldots, m$ are subadditive, non-decreasing and such that $D_{j}(0)=0, d_{i}(0)=\bar{d}_{i}(0)=\bar{d}_{i}(0)=d_{i}(0)$ $=0, \bar{\omega}_{s}(0)=\bar{\omega}_{s}(0)=0$, and, moreover:

$1^{\circ}\|F(x, u, v)-F(\bar{x}, \bar{u}, \bar{v})\| \leqslant D_{0}(|x-\bar{x}|)+\sum_{i=1}^{m} D_{i}\left(\left\|u_{i}-\bar{u}_{i}\right\|\right)+D_{m+1}(\|v-\bar{v}\|)$ for $x, \bar{x} \in G,\left\|u_{i}\right\|,\left\|\bar{u}_{i}\right\| \leqslant R_{i},\|v\|,\|\bar{v}\| \leqslant \tilde{r}$, where $\tilde{r}$ dx $\sup _{x \in G} \tilde{z}(x), \dot{R}_{i} \stackrel{\text { def }}{=}$ $\sup _{x \in G} L_{p_{i}}\left(H_{i}(x)\right) \sup _{x \in G} \bar{h}_{i}(x) \sup _{x \in G} \tilde{z}(x)+\sup _{x \in G} \bar{g}_{l}(x)$, and $\tilde{z}$ defined in Lemma 1 .

$2^{\circ}\left\|f_{i}(x, s, z)-f_{i}(\bar{x}, \bar{s}, \bar{z})\right\| \leqslant d_{i}(|x-\bar{x}|)+\bar{d}_{i}(|s-\bar{s}|)+\bar{d}_{i}(\|z-\bar{z}\|), i=1, \ldots, m$ for $x, \bar{x}, s, \bar{s} \in G,\|z\|,\|\bar{z}\| \leqslant \tilde{r}$.

$3^{\circ} \quad L_{n}\left(H_{i}(x+h)-H_{i}(x)\right) \leqslant \mathscr{d}_{i}(|h|)$ for $i \in A^{\prime}, x, x+h \in G$,

$L_{p_{i}}\left[H_{i}(x)-\left(-t_{i}(x, h)+H_{i}(x+h)\right)\right] \leqslant \mathcal{d}_{i}(|h|) \quad$ for $i \in B^{\prime}, x, x+h \in G$.

$4^{\circ}\left|t_{i}(x, h)\right| \leqslant \bar{\omega}_{i}(|h|)$ for $i \in B^{\prime},|h| \in\left[0, r_{0}\right]$, where $r_{0}$ is the diameter of the set $G$,

$$
\left|\alpha_{i}(x+h)-\alpha_{i}(x)\right| \leqslant \bar{\omega}_{i}(|h|) \quad \text { for } i \in B^{\prime}, x \in G,|h| \in\left[0, r_{0}\right] .
$$

Let $\tilde{\omega}_{i}(t)=\bar{\omega}_{i}\left(\bar{\omega}_{i}(t)\right), i \in B^{\prime}, t \in\left[0, r_{0}\right]$.

\section{A certain functional equation.}

LemMa 10. If

$1^{\circ}$ Assumption $\mathrm{H}_{5}$ and conditions $1^{\circ} ; 3^{\circ}$ from Assumption $\mathrm{H}_{4}$ are satisfied, 
$2^{\circ}$ the Lipschitz condition

$$
\|F(x, u, v)-F(x, u, \bar{v})\| \leqslant k(x)\|v-\bar{v}\|, \quad x \in G, v, \bar{v} \in B
$$

holds,

$3^{\circ} W \stackrel{\text { def }}{=}\{y: y \in C(G, B),\|y(x)\| \leqslant \tilde{z}(x)$ for $x \in G\}$,

then for any $y \in W$ there exists the unique $u(\cdot, y) \in W$ being a solution of the equation

$$
u(x)=F\left(x, \int_{H(x)} f(x, s, y(\alpha(s))) d s, u(\beta(x))\right), \quad x \in G .
$$

Proof. Put

$$
\begin{array}{r}
. u_{0}(x)=0, \quad u_{r+1}(x)=F\left(x, \int_{H(x)} f(x, s, y(\alpha(s))) d s, u_{r}(\beta(x))\right), \\
x \in G \quad r=0,1, \ldots
\end{array}
$$

We prove that

$$
\left\|u_{r}(x)\right\| \leqslant \tilde{z}(x), \quad x \in G, r=0,1,2, \ldots
$$

For $r=0$ this inequality is evidently satisfied. If we assume that $\left\|u_{r}(x)\right\|$ $\leqslant \tilde{z}(x)$ for $x \in G$, then

$$
\begin{aligned}
& \left\|u_{r+1}(x)\right\| \leqslant \| F\left(x, \int_{H(x)} f(x, s, y(\alpha(s))) d s, u_{r}(\beta(x))\right)- \\
& -F\left(x, \int_{H(x)} f(x, s, y(\alpha(s))) d s, 0\right)\|+\| F\left(x, \int_{H(x)} f(x, s, y(\alpha(s))) d s, 0\right) \| \\
& \quad \leqslant k(x) \tilde{z}(\beta(x))+K(x) \int_{H(x)} \tilde{z}(\alpha(s)) d s+\tilde{h}(x)=\tilde{z}(x)
\end{aligned}
$$

for $x \in G$. Now we obtain (38) by induction.

Next we prove that $u_{r}$ are continuous in $G$. Since $u_{0}$ is continuous in $G$, it is sufficient to prove that the continuity of $u_{r}$ implies the continuity of $u_{r+1}$. Let

$$
\tilde{R}_{1}=\max _{j}\left[\sup _{x \in G} \bar{h}_{j}(x) \sup _{x \in G}\|y(x)\|+\sup _{x \in G} \bar{g}_{j}(x)\right]
$$

By Assumption $\mathrm{H}_{5}$ we have

$$
\begin{aligned}
&\left\|\dot{u}_{r+1}(x+h)-u_{r+1}(x)\right\| \\
& \leqslant D_{0}(|h|)+\sum_{j=1}^{m} D_{j}\left(\|_{H_{j}(x+h)} f_{j}\left(x+h, s, y\left(\dot{\alpha}_{j}(s)\right)\right)(d s)_{p_{j}}-\right. \\
&\left.\quad-\int_{H_{j}(x)} f\left(x, s, y\left(\alpha_{j}(s)\right)\right)(d s)_{p_{j}} \|\right)+D_{m+1}\left(\left\|u_{r}(\beta(x+h))-u_{r}(\beta(x))\right\|\right) .
\end{aligned}
$$


Now for $j \in A^{\prime}$ we get, writing $d s$ instead of $(d s)_{n}$

(40)

$$
\begin{aligned}
& \quad\left\|\int_{H_{j}(x+h)} f_{j}\left(x+h, s, y\left(\alpha_{j}(s)\right)\right) d s-\int_{H_{j}(x)} f_{j}\left(x, s, y\left(\alpha_{j}(s)\right)\right) d s\right\| \\
& \leqslant \quad \int_{H_{j}(x+h) \cap H_{j}(x)}\left\|f_{j}\left(x+h, s, y\left(\alpha_{j}(s)\right)\right)-f_{j}\left(x, s, y\left(\alpha_{j}(s)\right)\right)\right\| d s+\int_{H_{j}(x+h)-H_{j}(x)} \tilde{R}_{1} d s \\
& \leqslant L_{n}(G) d_{j}(|h|)+\tilde{R}_{1} d_{j}(|h|) .
\end{aligned}
$$

If for $j \in B^{\prime}$ we define the sets $H_{j}^{0}(x, h), H_{j}^{1}(x, h), H_{j}^{2}(x, h), H_{j}^{3}(x, h)$ by

$$
\begin{aligned}
& H_{j}^{0}(x, h)=H_{j}(x)-\left(-t_{j}(x, h)+H_{j}(x+h)\right), \\
& H_{j}^{1}(x, h)=\left(-t_{j}(x, h)+H_{j}(x+h)\right) \cap H_{j}(x), \\
& H_{j}^{2}(x, h)=\left(-t_{j}(x, h)+H_{j}(x+h)\right)-H_{j}(x), \\
& H_{j}^{3}(x, h)=H_{j}(x)-\left(-t_{j}(x, h)+H_{j}(x+h)\right),
\end{aligned}
$$

then

(42)

$$
\begin{array}{ll} 
& \left\|\int_{H_{j}(x+h)} f_{j}\left(x+h, s, y\left(\alpha_{j}(s)\right)\right)(d s)_{p_{j}}-\int_{H_{j}(x)} f_{j}\left(x, s, y\left(\alpha_{j}(s)\right)\right)(d s)_{p_{j}}\right\| \\
\leqslant & \int_{H_{j}^{1}(x, h)}\left\|f_{j}\left(x+h, s+t_{j}(x, h), y\left(\alpha_{j}\left(s+t_{j}(x, h)\right)\right)\right)-f_{j}\left(x, s, y\left(\alpha_{j}(s)\right)\right)\right\|(d s)_{p_{j}}+ \\
& +\int_{H_{j}^{2}(x, h)}\left\|f_{j}\left(x+h, s+t_{j}(x, h), y\left(\alpha_{j}\left(s+t_{j}(x, h)\right)\right)\right)\right\|(d s)_{p_{j}}+ \\
& +\int_{H_{j}^{3}(x, h)}\left\|f_{j}\left(x, s, y\left(\alpha_{j}(s)\right)\right)\right\|(d s)_{p_{j}} \\
\leqslant & L_{p_{j}}\left(H_{j}(x)\right)\left[d_{j}(|h|)+\bar{d}_{j}\left(\tilde{\omega}_{j}(|h|)\right)+\right. \\
& \left.+\bar{d}_{j}\left(\sup _{s \in G}\left\|y\left(\alpha_{j}\left(s+t_{j}(x, h)\right)\right)-y\left(\alpha_{j}(s)\right)\right\|\right)\right]+\int_{H_{j}^{0}(x, h)}^{\int} \tilde{R}_{1}(d s)_{p_{j}} \\
\leqslant & L_{p_{j}}\left(H_{j}(x)\right)\left[d_{j}(|h|)+\bar{d}_{j}\left(\tilde{\omega}_{j}(|h|)\right)+\bar{d}_{j}\left(\tilde{d}\left(\tilde{\omega}_{j}(|h|)\right)\right)+\tilde{R}_{1} \dot{d}_{j}(|h|)\right]
\end{array}
$$

where $\tilde{d}$ is a modulus of continuity for the function $y$. It follows from (39), (40), (42) and from the continuity of $y, u_{r}, \alpha_{j}, \beta$ that $u_{r+1}$ is continuous. We put

$$
z_{0}(x)=\tilde{z}(x) \quad \text { for } x \in G, \quad z_{r}(x)=k(x) z_{r-1}(\beta(x)), \quad r=1,2, \ldots, x \in G .
$$

By induction we get

$$
z_{r}(x)=k^{(r)}(x) \tilde{z}\left(\beta^{(r)}(x)\right), \quad r=1,2, \ldots, x \in G .
$$

In virtue of condition (9) of Lemma 1 it follows from (43) that

$$
\lim _{r \rightarrow \infty} z_{r}(x)=0
$$


and the convergence is uniform with respect to $x \in G$. Further, we get easily

$$
\left\|u_{r+p}(x)-u_{r}(x)\right\| \leqslant z_{r}(x), \quad x \in G, r, p=0,1,2, \ldots
$$

Indeed, from (37) and (38) it follows that (45) is satisfied for $r=0$, $p=0,1,2, \ldots, x \in G$. If we assume that (45) holds for a fixed $r$ and $p=0,1,2, \ldots, x \in G$, then

$$
\begin{aligned}
\left\|u_{r+1+p}(x)-u_{r+1}(x)\right\| & \leqslant k(x)\left\|u_{r+p}(\beta(x))-u_{r}(\beta(x))\right\| \\
& \leqslant \dot{k}(x) z_{r}(\beta(x))=z_{r+1}(x) .
\end{aligned}
$$

Now we obtain (45) by induction.

By (37), (44), (45) we infer that the sequence $\left\{u_{r}\right\}$ is uniformly convergent in $G$ to the solution $\bar{u}$ of equation (36). Since the sequence $\left\{u_{r}\right\}$ is uniformly convergent in $G$ and $u_{r} \in C(G, B)$, we conclude by (38) that $\bar{u} \in W$.

To prove that the solution $\bar{u}$ of (36) is unique in $W$, let us suppose that there exists another solution $\bar{u} \neq \bar{u}$ and $\bar{u} \in W$. Now, from (35) we have

$$
\|\bar{u}(x)-\bar{u}(x)\| \leqslant k(x)\|\bar{u}(\beta(x))-\bar{u}(\beta(x))\|, \quad x \in G,
$$

and by induction we get

$$
\|\bar{u}(x)-\bar{u}(x)\| \leqslant k^{(r)}(x)\left\|\bar{u}\left(\beta^{(r)}(x)\right)-\bar{u}\left(\beta^{(r)}(x)\right)\right\|, \quad r=0,1,2, \ldots
$$

Since

$$
\begin{array}{ll}
k^{(r)}(x)\left\|\bar{u}\left(\beta^{(r)}(x)\right)\right\| \leqslant k^{(r)}(x) \tilde{z}\left(\beta^{(r)}(x)\right), & r=0,1,2, \ldots, x \in G, \\
k^{(r)}(x)\left\|\overline{\bar{u}}\left(\beta^{(r)}(x)\right)\right\| \leqslant k^{(r)}(x) \tilde{z}\left(\beta^{(r)}(x)\right), & r=0,1,2, \ldots, x \in G,
\end{array}
$$

and

$$
\lim _{r \rightarrow \infty} k^{(r)}(x) \tilde{z}\left(\beta^{(r)}(x)\right)=0 \quad \text { uniformly with respect to } x \in G,
$$

we infer by (46) that $\bar{u}=\bar{u}$. This contradiction proves the uniqueness of $\bar{u}$ in $W$.

3. Further assumptions. We introduce

Assumption $\mathrm{H}_{6}$. Suppose that

$x \in G, \delta_{j} \in R_{+}$,

$$
1^{\circ} m_{0}\left(x, \delta_{1}, \delta_{2}, \ldots, \delta_{m}\right)=\sum_{i=0}^{\infty} k^{(i)}(x) \sum_{j=1}^{m} D_{j}\left(\delta_{j} L_{p_{j}}\left(G_{j}\left(\beta^{(i)}(x)\right)\right)\right)<+\infty \text { for }
$$

$2^{\circ}$ the function $m_{0}$ is continuous with respect to $\left(x, \dot{\delta}_{1}, \ldots, \delta_{m}\right) \in G \times R_{+}^{m}$.

Remark 5. It is obvious that Assumption $\mathrm{H}_{6}$ is fulfilled if, for instance, $k(x) \leqslant \bar{k}<1$ for $x \in G$. If condition $1^{\circ}$ of $\mathrm{H}_{6}$ is satisfied and the functions $k, \beta, \alpha_{j}$ are non-decreasing in $G$, then condition $2^{\circ}$ of $\mathrm{H}_{6}$ is fulfilled. 
Remark 6. If the functions $\varphi_{i}$ and $\beta$ satisfy conditions $2^{\circ}$ and $3^{\circ}$ of Lemma 4, respectively, and

$$
\begin{gathered}
k(x) \leqslant \bar{k}=\text { const, } \quad D_{j}(t) \leqslant D t, \quad D=\text { const, } \quad j=1, \ldots, m, \\
\bar{k} \prod_{s \in \bar{o}_{j}} \bar{\beta}_{s}<1, \quad j=1,2, \ldots, m,
\end{gathered}
$$

then Assumption $\mathrm{H}_{6}$ is fulfilled (see the proof of Lemma 4).

We adopt the following notation:

$$
\begin{gathered}
\bar{K}=\left(K_{k_{0}+1}, \ldots, K_{m}\right), \quad \bar{L}(G(x))=\left(L_{p_{k_{0}+1}}\left(G_{k_{0}+1}(x)\right), \ldots, L_{p_{m}}\left(G_{m}(x)\right)\right), \\
\bar{K}(x) \cdot \bar{L}(G(x))=\sum_{j=k_{0}+1}^{m} K_{j}(x) \cdot L_{p_{j}}\left(G_{j}(x)\right), \\
\int_{H(x)} d(\alpha(s), \tilde{\omega}(t)) d s=\left(\int_{H_{k_{0}+1}(x)} d\left(\alpha_{k_{0}+1}(s), \tilde{\omega}_{k_{0}+1}(t)\right)(d s)_{p_{k_{0}+1}}, \ldots\right. \\
\left.\ldots, \int_{H_{m}^{(x)}} d\left(\alpha_{m}(s), \bar{\omega}_{m}(t)\right)(d s)_{p_{m}}\right), \\
\bar{K}(x) \int_{\bar{H}(x)} d(\alpha(s), \bar{\omega}(t)) d s=\sum_{j=k_{0}+1}^{m} K_{j}(x) \int_{H_{j}(x)} d\left(\alpha_{j}(s), \tilde{\omega}_{j}(t)\right)(d s)_{p_{j}},
\end{gathered}
$$

where $\tilde{\omega}_{i}$ are real-valued functions of one variable.

We introduce

Assumption $\mathrm{H}_{7}$. Suppose that

$1^{\circ}|\beta(x+h)-\beta(x)| \leqslant \omega(|h|)$, for $x, x+h \in G$, where $\omega \in C\left(R_{+}, R_{+}\right)$is subadditive and non-decreasing and

$$
\omega(0)=0, \quad \bar{\omega}=\left(\tilde{\omega}_{k_{0}+1}, \ldots, \tilde{\omega}_{m}\right) \in C\left(R_{+}, R_{+}^{m-k_{0}}\right),
$$

$\tilde{\omega}_{i}$ are subadditive and non-decreasing, and $\tilde{\omega}_{i}(0)=0$,

$2^{\circ}$ there is given a function $p$ such that

(a) $p \in C\left(G \times\left[0, r_{0}\right], R_{+}\right)$, where $r_{0} \in R_{+}$is defined in $4^{\circ}$ of $\mathrm{H}_{5}$,

(b) $p$ is non-decreasing and subadditive with respect to the last variable,

(c) $p(x, 0)=0$ for $x \in G$,

$$
3^{\circ} \bar{m}(x, t)=\sum_{i=0}^{\infty} k^{(i)}(x) p\left(\beta^{(i)}(x), \omega^{(i)}(t)\right)<+\infty \quad \text { for } \quad(x, t) \in G \times\left[0, r_{0}\right] \text {, }
$$

where $\omega^{(0)}(t)=t, \omega^{(i+1)}(t)=\omega\left(\omega^{(i)}(t)\right), i=0,1,2, \ldots, t \in\left[0, r_{0}\right]$,

$4^{0} \bar{M}(x)=\sum_{i=0}^{\infty} k^{(i)}(x)\left[\bar{K}\left(\beta^{(i)}(x)\right) \cdot \bar{L}\left(G\left(\beta^{(i)}(x)\right)\right)\right]<+\infty, x \in G$,

$5^{\circ} \bar{m} \in C\left(G \times\left[0, r_{0}\right], R_{+}\right), \bar{M} \in C\left(G, R_{+}\right)$, 
$6^{\circ}$ the function

$$
\bar{M}(x)=\sum_{i=0 .}^{\infty} k^{(i)}(x)\left[\sum_{j=k_{0}+1}^{m} K_{j}\left(\beta^{(i)}(x)\right) L_{p_{j}}\left(G_{j}\left(\beta^{(i)}(x)\right)\right) \cdot\left(\prod_{s \in \bar{\sigma}_{j}} x_{s}\right)^{-1}\right]
$$

is bounded in $G$.

We have the following

Lemma 11. If Assumption $\mathrm{H}_{7}$ and condition $2^{\circ}$ of Assumption $\mathrm{H}_{1}$ are satisfied, then:

$1^{\circ}$ There exists a solution $\mathbb{d} \in C\left(G \times\left[0, r_{0}\right], R_{+}\right)$of the equation

$$
\begin{aligned}
d(x, t)= & \sum_{i=0}^{\infty} k^{(i)}(x) p\left(\beta^{(i)}(x), \omega^{(i)}(t)\right)+ \\
& +\sum_{i=0}^{\infty} k^{(i)}(x)\left[\bar{K}\left(\beta^{(i)}(x)\right) \cdot \int_{\bar{H}\left(\beta^{(i)}(x)\right)} d\left(\alpha(s), \bar{\omega}\left(\omega^{(i)}(t)\right)\right) d s\right], \\
& (x, t) \in G \times\left[0, r_{0}\right] .
\end{aligned}
$$

The solution $d$ of (47) is unique in the class $M\left(G \times\left[0, r_{0}\right], R_{+}\right)$of nonnegative upper-semicontinuous functions defined on $G \times\left[0, r_{0}\right]$. The function $d$ is non-decreasing and subadditive with respect to the last variable and $\partial(x, 0)=0$ for $x \in G$.

$2^{\circ}$ The function $d$ is a solution of the equation

$$
\begin{aligned}
d(x, t)=\bar{K}(x) \int_{\tilde{H}(x)} d(\alpha(s), \tilde{\omega}(t)) d s+k(x) d(\beta(x), \omega(t))+p(x, t), & (x, t) \in G \times\left[0, r_{0}\right] .
\end{aligned}
$$

Moreover, this solution is unique in the class $\tilde{M}\left(G \times\left[0, r_{0}\right], R_{+}, d\right)$, where $\tilde{M}\left(G \times\left[0, r_{0}\right], R_{+}, \tilde{d}\right)=\left\{z: z \in M\left(G \times\left[0, r_{0}\right], R_{+}\right)\right.$, inf $[c: z(x, t) \leqslant c d(x, t)]$ $<+\infty\}$.

The proof of this Lemma is similar to the proof of assertions $1^{\circ}, 2^{\circ}$ of Lemma 1.

4. Properties of the operator $U$. Let $\tilde{W}=\{y: y \in C(G, B),\|y(x)\| \leqslant \tilde{z}(x)$, $\|y(x+h)-y(x)\| \leqslant \mathcal{d}(x,|h|)\}$, where the functions $\tilde{z}$ and $\mathcal{J}$ are defined by Lemma 1 and Lemma 11, respectively. We consider the operator $U$ defined by the formula $U y=u(\cdot, y)$, where $u(\cdot, y)$ is the solution of functional equation (36).

We have

Lemma 12. If Assumptions $\mathrm{H}_{5}, \mathrm{H}_{6}$, conditions $1^{\circ}$ and $3^{\circ}$ from $\dot{\mathrm{H}_{4}}$ and the Lipschitz condition (35) are satisfied, then the operator $U$ is continuous in the set $W$. 
Proof. Let $y_{1}, y_{2} \in W, u_{1}=u\left(\cdot, y_{1}\right), u_{2}\left(\cdot, y_{2}\right), v(x)=\left\|u_{1}(x)-u_{2}(x)\right\|$. Then we have for $x \in G$

$$
\begin{aligned}
v(x)= & \| F\left(x, \int_{H(x)} f\left(x, s, y_{1}(\alpha(s))\right) d s, u_{1}(\beta(x))\right)- \\
& -F\left(x, \int_{H(x)} f\left(x, s, y_{2}(\alpha(s))\right) d s, u_{2}(\beta(x))\right) \| \\
\leqslant & \sum_{j=1}^{m} D_{j}\left(\left\|\int_{H_{j}(x)}\left[f_{j}\left(x, s, y_{1}\left(\alpha_{j}(s)\right)\right)-f_{j}\left(x, s, y_{2}\left(\alpha_{j}(s)\right)\right)\right](d s)_{p_{j}}\right\|\right)+ \\
& +k(x)\left\|u_{1}(\beta(x))-u_{2}(\beta(x))\right\| \\
\leqslant & \sum_{j=1}^{m} D_{j}\left(\int_{H_{j}(x)} \bar{d}_{j}\left(\left\|y_{1}\left(\alpha_{j}(s)\right)-y_{2}\left(\alpha_{j}(s)\right)\right\|\right)(d s)_{p_{j}}\right)+k(x) v(\beta(x)) .
\end{aligned}
$$

Let $\delta_{j}=\bar{d}_{j}\left(\sup _{s \in G}\left\|y_{1}(s)-y_{2}(s)\right\|\right)$. Then we have the inequality

$$
v(x) \leqslant \sum_{j=1}^{m} D_{j}\left(\delta_{j} L_{p_{j}}\left(G_{j}(x)\right)\right)+k(x) v(\beta(x)), \quad x \in G
$$

and we get by induction

(49) $\quad v(x) \leqslant \sum_{i=0}^{r-1} k^{(i)}(x)\left[\sum_{j=1}^{m} D_{j}\left(\delta_{j} L_{p_{j}}\left(G_{j}\left(\beta^{(i)}(x)\right)\right)\right)\right]+k^{(r)}(x) v\left(\beta^{(r)}(x)\right)$,

$$
x \in G, r=1,2, \ldots
$$

Since

$$
0 \leqslant k^{(r)}(x) v\left(\beta^{(r)}(x)\right) \leqslant 2 k^{(r)}(x) \tilde{z}\left(\beta^{(r)}(x)\right), \quad r=0,1,2, \ldots, x \in G
$$

and

$$
\lim _{r \rightarrow \infty} k^{(r)}(x) \tilde{z}\left(\beta^{(r)}(x)\right)=0 \quad \text { uniformly with respect to } x \in G
$$

we get, making $r \rightarrow \infty$ in (49), that

$$
v(x) \leqslant \sum_{i=0}^{\infty} k^{(i)}(x)\left[\sum_{j=1}^{m} D_{j}\left(\delta_{j} L_{p_{j}}\left(G_{j}\left(\beta^{(i)}(x)\right)\right)\right)\right]=m_{0}\left(x, \delta_{1}, \ldots, \delta_{m}\right) .
$$

In view of the continuity of the function $m_{0}$ we conclude the assertion of Lemma 12. 
Lemma 13. Suppose that:

$1^{\circ}$ Assumptions $\mathbf{H}_{4}, \mathbf{H}_{5}, \mathbf{H}_{6}$ are satisfied and Assumption $\mathrm{H}_{7}$ is fulfilled for $p, \bar{K}$ defined by the relations

$$
\begin{aligned}
p(x, t)=D_{0}(t) & +\sum_{j=1}^{k_{0}} \dot{D}_{j}\left(L_{n}(G) d_{j}(t)+P_{j} \partial_{j}(t)\right)+ \\
& +\sum_{j=k_{0}+1}^{m} l_{j}(x)\left[L_{p_{j}}\left(H_{j}(x)\right)\left(d_{j}(t)+\bar{d}_{j}\left(\bar{\omega}_{j}(t)\right)\right)+P_{j} \partial_{j}(t)\right]
\end{aligned}
$$

where $P_{j}=\sup _{x \in G} \bar{h}_{j}(x) \sup _{x \in G} \tilde{z}(x)+\sup _{x \in G} \bar{g}_{j}(x)$,

$$
\bar{K}(x)=\left(l_{k_{0}+1}(x) \bar{l}_{k_{0}+1}(x), \ldots, l_{m}(x) \bar{l}_{m}(x)\right), \quad x \in G
$$

$2^{\circ}$ For $x, x+h \in G$ we have

$$
\lim _{r \rightarrow \infty} k^{(r)}(x) \tilde{z}\left(\beta^{(r)}(x+h)\right)=0 \quad \text { uniformly with respect to } x, x+h \in G
$$

Under these assumptions the operator $U$ maps $\tilde{W}$ into itself.

Proof. In virtue of Lemma 10 it follows that for each $y \in \tilde{W}$ the function $U y$ satisfies the condition $\|(U y)(x)\| \leqslant \tilde{z}(x)$ for $x \in G$. To prove that $U y \in \tilde{W}$ for $y \subset \tilde{W}$, it is sufficient to show that $\|(U y)(x+h)-(U y)(x)\| \leqslant \mathcal{d}(x,|h|)$ for $x, x+h \in G$.

Let us suppose that $y \in \tilde{W}$ and $u(x)=(U y)(x)$. We show that

$$
\|u(x+h)-u(x)\| \leqslant d(x,|h|), \quad x, x+h \in G .
$$

For $j \in A^{\prime}$ we get, writing $d s$ instead of $(d s)_{n}$ for simplicity,

$$
\begin{gathered}
\left\|\int_{H_{j}(x+h)} f_{j}\left(x+h, s, y\left(\alpha_{j}(s)\right)\right) d s-\int_{H_{j}(x)} f_{j}\left(x, s, y\left(\alpha_{j}(s)\right)\right) d s\right\| \\
\leqslant \int_{H_{j}(x+h) \cap H_{j}(x)}\left\|f_{j}\left(x+h, s, y\left(\alpha_{j}(s)\right)\right)-f_{j}\left(x, s, y\left(\alpha_{j}(s)\right)\right)\right\| d s+ \\
\quad+\int_{H_{j}(x+h)-H_{j}(x)}\left[\bar{h}_{j}(x+h)\left\|y\left(\alpha_{j}(s)\right)\right\|+\bar{g}_{j}(x+h)\right] d s+ \\
\quad+\int_{H_{j}(x)-H_{j}(x+h)}\left[\bar{h}_{j}(x)\left\|y\left(\alpha_{j}(s)\right)\right\|+\bar{g}_{j}(x)\right] d s \\
\leqslant L_{n}(G) d_{j}(|h|)+P_{j} \bar{d}_{j}(|h|) .
\end{gathered}
$$

In the case $j \in B^{\prime}$ we define the sets $H_{j}^{k}(x, h), k=0,1,2,3$, by (41) and note that integration over the set $H_{j}(x+h)$ is equivalent to integration over $-t_{j}(x, h)+H_{j}(x+h)$ if one replaces the variable $s$ by $s+t_{j}(x, h)$. 
In this way we arrive at

$$
\begin{aligned}
& \left\|\int_{H_{j}(x+h)} f_{j}\left(x+h, s, y\left(\alpha_{j}(s)\right)\right)(d s)_{p_{j}}-\int_{H_{j}(x)} f_{j}\left(x, s, y\left(\alpha_{j}(s)\right)\right)(d s)_{p_{j}}\right\| \\
& =\| \int_{H_{j}^{1}(x, h)} f_{j}\left(x+h, s+t_{j}(x, h), y\left(\alpha_{j}\left(s+t_{j}(x, h)\right)\right)\right)(d s)_{p_{j}}- \\
& -\int_{H(x, h)} f_{j}\left(x, s, y\left(\alpha_{j}(s)\right)\right)(d s)_{p_{j}}+ \\
& +\int_{H_{j}^{2}(x, h)} f_{j}\left(x+h, s+t_{j}(x, h), y_{.}\left(\alpha_{j}\left(s+t_{j}(x, h)\right)\right)\right)(d s)_{p_{j}}- \\
& -\int_{H_{j}^{3}(x, h)} f_{j}\left(x, s, y\left(\alpha_{j}(s)\right)\right)(d s)_{p_{j}} \| \\
& \leqslant \int_{H j(x, h)}\left\|f_{j}\left(x+h, s+t_{j}(x, h), y\left(\alpha_{j}\left(s+t_{j}(x, h)\right)\right)\right)-f_{j}\left(x, s, y\left(\alpha_{j}(s)\right)\right)\right\|(d s)_{p_{j}}+ \\
& +\int_{H_{j}^{2}(x, h)} P_{j}(d s)_{p_{j}}+\int_{H_{j}^{3}(x, h)} P_{j}(d s)_{p_{j}} \\
& \leqslant \int_{H_{j}^{\prime}(x, h)}\left[d_{j}(|h|)+\bar{d}_{j}\left(\bar{\omega}_{j}(|h|)\right)+\bar{l}_{j}(x)\left\|y\left(\alpha_{j}\left(s+t_{j}(x, h)\right)\right)-y\left(\alpha_{j}(s)\right)\right\|\right](d s)_{p_{j}}+ \\
& +\int_{H \rho(x, h)} P_{j}(d s)_{p_{j}} \\
& \leqslant L_{p_{j}}\left(H_{j}(x)\right)\left[d_{j}(|h|)+\bar{d}_{j}\left(\bar{\omega}_{j}(|h|)\right)\right]+ \\
& +\bar{l}_{j}(x) \int_{H_{j}(x)}\left\|y\left(\alpha_{j}\left(s+t_{j}(x, h)\right)\right)-y\left(\alpha_{j}(s)\right)\right\|(d s)_{p_{j}}+P_{j} d_{j}(|h|) .
\end{aligned}
$$

It follows from Assumption $\mathrm{H}_{5}$ and from the above estimates that

$$
\begin{aligned}
\|u(x+h)-u(x)\|= & \| F\left(x+h, \int_{H(x+h)} f(x+h, s, y(\alpha(s))) d s, u(\beta(x+h))\right)- \\
& -F\left(x, \int_{H(x)} f(x, s, y(\alpha(s))) d s, u(\beta(x))\right) \| \\
\leqslant & D_{0}(|h|)+\sum_{j=1}^{k_{0}} D_{j}\left[\| \int_{H_{j}(x+h)} f_{j}\left(x+h, s, y\left(\alpha_{j}(s)\right)\right)(d s)_{p_{j}}-\right. \\
& \left.-\int_{H_{j}(x)} f_{j}\left(x, s, y\left(\alpha_{j}(s)\right)\right)(d s)_{p_{j}} \|\right]+ \\
& +\sum_{j=k_{0}+1}^{m} l_{j}(x) \| \int_{H_{j}(x+h)} f_{j}\left(x+h, s, y\left(\alpha_{j}(s)\right)\right)(d s)_{p_{j}}- \\
& -\int_{H_{j}(x)} f_{j}\left(x, s, y\left(\alpha_{j}(s)\right)\right)(d s)_{p_{j}}\|+k(x)\| u(\beta(x+h))-u(\beta(x)) \|
\end{aligned}
$$




$$
\begin{aligned}
& \leqslant D_{0}(|h|)+\sum_{j=1}^{k_{0}} D_{j}\left[L_{n}(G) d_{j}(|h|)+P_{j} d_{j}(|h|)\right]+ \\
& \quad+\sum_{j=k_{0}+1}^{m} l_{j}(x)\left\{L_{p_{j}}\left(H_{j}(x)\right)\left[d_{j}(|h|)+\bar{d}_{j}\left(\bar{\omega}_{j}(|h|)\right)\right]+P_{j} d_{j}(|h|)\right\}+ \\
& \quad+\sum_{j=k_{0}+1}^{m} l_{j}(x) \cdot \bar{l}_{j}(x) \int_{H_{j}(x)}\left\|y\left(\alpha_{j}\left(s+t_{j}(x, h)\right)\right)-y\left(\alpha_{j}(s)\right)\right\|(d s)_{p_{j}}+ \\
& \quad+k(x)\|u(\beta(x+h))-u(\beta(x))\|^{.} \\
& \leqslant p(x,|h|)+\sum_{j=k_{0}+1}^{m} K_{j}(x) \int_{H_{j}(x)}\left\|y\left(\alpha_{j}\left(s+t_{j}(x, h)\right)\right)-y\left(\alpha_{j}(s)\right)\right\|(d s)_{p_{j}}+ \\
& \quad+k(x)\|u(\beta(x+h))-u(\beta(x))\| .
\end{aligned}
$$

Since $\|y(x+h)-y(x)\| \leqslant d(x,|h|)$, we have

$$
\left\|y\left(\alpha_{j}\left(s+t_{j}(x, h)\right)\right)-y\left(\alpha_{j}(s)\right)\right\| \leqslant d\left(\alpha_{j}(s), \tilde{\omega}_{j}(|h|)\right)
$$

and consequently

$$
\begin{aligned}
\|u(x+h)-u(x)\| \leqslant p(x,|h|)+\bar{K}(x) \int_{\overline{\boldsymbol{H}}(\mathbf{x})} d(\alpha(s), \tilde{\omega}(|h|)) d s+ \\
\quad+k(x)\|u(\beta(x+h))-u(\beta(x))\| .
\end{aligned}
$$

The last inequality implies the following:

$$
\begin{aligned}
& \|u(x+h)-u(x)\| \leqslant \sum_{i=0}^{r-1} k^{(i)}(x) p\left(\beta^{(i)}(x), \mid \beta^{(i)}(x+h)-\beta^{(i)}(x) \|\right)+ \\
& +\sum_{i=0}^{r-1} k^{(i)}(x)\left[\bar{K}\left(\beta^{(i)}(x)\right) \int_{\bar{H}\left(\beta^{(i)}(x)\right)} \tilde{\partial}\left(\alpha(s), \tilde{\omega}\left(\left|\beta^{(i)}(x+h)-\beta^{(i)}(x)\right|\right)\right) d s\right]+ \\
& +k^{(r)}(x)\left\|u\left(\beta^{(r)}(x+h)\right)-u\left(\beta^{(r)}(x)\right)\right\|, \quad x, x+h \in G, r=1,2, \ldots
\end{aligned}
$$

It follows from the inequalities

$$
\begin{aligned}
& k^{(r)}(x)\left\|u\left(\beta^{(r)}(x+h)\right)-u\left(\beta^{(r)}(x)\right)\right\| \leqslant k^{(r)}(x)\left\|u\left(\beta^{(r)}(x)\right)\right\|+k^{(r)}(x)\left\|u\left(\beta^{(r)}(x+h)\right)\right\| \\
& \quad \leqslant k^{(r)}(x) \tilde{z}\left(\beta^{(r)}(x)\right)+k^{(r)}(x) \tilde{z}\left(\beta^{(r)}(x+h)\right), \quad x, x+h \in G, r=0,1,2, \ldots,
\end{aligned}
$$

and from conditions (9) and (52) that

$$
\lim _{r \rightarrow \infty} k^{(r)}(x)\left\|u\left(\beta^{(r)}(x+h)\right)-u\left(\beta^{(r)}(x)\right)\right\|=0 \quad \text { uniformly in } G .
$$

By induction we easily obtain

$$
\left|\beta^{(i)}(x+h)-\beta^{(i)}(x)\right| \leqslant \omega^{(i)}(|h|), \quad x, x+h \in G, i=0,1,2, \ldots
$$


Now, from (55), (56) and by the definition of $d$, we have, letting $r \rightarrow \infty$ in (54),

$$
\begin{aligned}
\|u(x+h)-u(x)\| & \leqslant \sum_{i=0}^{\infty} k^{(i)}(x) p\left(\beta^{(i)}(x), \omega^{(i)}(|h|)\right)+ \\
& +\sum_{i=0}^{\infty} k^{(i)}(x)\left[\bar{K}\left(\beta^{(i)}(x)\right) \int_{H(\beta(i)(x))} \partial\left(\alpha(s), \tilde{\omega}\left(\omega^{(i)}(|h|)\right)\right) d s\right]=\partial(x,|h|),
\end{aligned}
$$

which completes the proof of (53).

Remark 7. If the functions $k, \tilde{h}, K, \beta$ are non-decreasing in $G$ and $H_{j}(x) \subset H_{j}(\bar{x})$ for $x<\bar{x}, x, \bar{x} \in G, j=1,2, \ldots, m$, then assumption $2^{\circ}$ of Lemma 13 is satisfied. This fact follows from assertion $4^{\circ}$ of Lemma 1 and from (9).

Now, we have the following

THEOREM 3. Suppose that:

$1^{\circ}$ Assumptions $\mathrm{H}_{4}, \mathrm{H}_{5}, \mathrm{H}_{6}$ are satisfied,

$2^{\circ}$ Assumption $\mathrm{H}_{7}$ is fulfilled for $p, \bar{K}$ defined by relations (50), (51),

$3^{\circ}$ condition (52) of Lemma 13 holds.

Under these assumptions equation (2) has at least one solution $\tilde{u} \in \tilde{W}$.

Proof. It follows from Lemmas $10,12,13$ that the continuous operator $U$ maps the compact and convex set $\tilde{W} \subset C(G, B)$ into itself. By the Schauder fixed point theorem there exists at least one solution $\tilde{u} \in \tilde{W}$ of equation (2).

\section{LEMMA 14. If} const,

$1^{\circ} k(x) \leqslant \bar{k}=$ const, $\bar{K}(x)=\left(K_{k_{0}+1}(x), \ldots, K_{m}(x)\right) \leqslant\left(\bar{K}_{k_{0}+1}, \ldots, \bar{K}_{m}\right)=$

$2^{\circ}$ the functions $\varphi_{i}$ and $\beta$ satisfy conditions $2^{\circ}$ and $3^{\circ}$, respectively, of Lemma 4,

$3^{\circ}$ there exist constants $\omega_{0}$ and $D$ such that $D_{i}(t), d_{j}(t), d_{j}(t), \bar{d}_{r}(t)$, $\bar{\omega}_{r}(t) \leqslant D t, i=1, \ldots, k_{0}, j=1, \ldots, m, r=k_{0}+1, \ldots, m$, and $\omega(t) \leqslant \omega_{0} \cdot t$,

$4^{\circ} \vec{k} \prod_{s \in \sigma_{j}} \bar{\beta}_{s}<1$ for $j=k_{0}+1, \ldots, m$,

$5^{\circ} \bar{k} \cdot \omega_{0}<1$

then conditions $3^{\circ}-6^{\circ}$ of Assumption $\mathrm{H}_{7}$ are fulfilled.

The proof of this lemma is similar to the proof of Lemma 4. Using this Lemma we can easily formulate a theorem which is more effective than Theorem 3. 


\section{References}

[1] T. Jankowski and M. Kwapisz, On the existence and uniqueness of solutions of systems of differential equations with a deviated argument, Ann. Polon. Math. 26(1972), p. 253-277.

[2] Z. Kamont and M. K wapisz, On the Cauchy problem for differential-delay equations in a Banach space, Math. Nachr. 74 (1976), p. 173-190.

[3] $\mathrm{M}$. Kwapisz, On the existence and uniqueness of solutions of a certain integral-functional equation, Ann. Polon. Math. 31 (1975), p. 23-41.

[4] - and J. Turo, On the existence and uniqueness of solutions of Darboux problem for partial differential-functional equations in a Banach space, ibidem 29 (1974), p. 89-118.

[5] - - On the existence and convergence of successive approximations for some functional equations in a Banach space, J. Diff. Eq. 16(1974), p. 289-318.

[6] - - On the existence and uniqueness of solutions of Darboux problem for partial differential-functional equations, Colloq. Math. 29 (1974), p. 279-302.

[7] - Existence, uniqueness and successive approximations for a class of integral-functional equations, Aeq. Math. 14 (1976), p. 303-323.

[8] - - On some integral-functional equations with an unknown function of $n$ variables, Bull. Acad. Polon. Sci., Sér. sci. math, astr. et phys. 22 (1974), p. 1001-1009.

[9] - - Some integral-functional equations, Funk. Ekv. 18 (1975), p. 107-162.

[10] - - On a class of functional-integral equations treated by the comparative method, (unpublished).

[11] J. Turo, $O$ istnieniu $i$ jednoznaczności rozwiqzań oraz zbieżności ciagu prostych $i$ przybliżonych iteracji dla równań calkowo-funkcyjnych z niewiadoma funkcja wielu zmiennych (in Polish), doctor's thesis, Faculty of Math., Phys. and Chem., University of Gdanisk 1974.

[12] W. Walter, On nonlinear Volterra integral equations in several variables, J. Math. Mech. 16 (9) (1967), p. 967-985.

[13] - Differential and integral inequalities, Springer-Verlag, Berlin, Heidelberg, New York 1970.

[14] T. Ważewski, Sur une procédé de prouver la convergence des approximations successives sans utilisation des. séries de comparison, Bull. Acad. Polon. Sci., Sér. sci. math., astr. et phys. 8 (1) (1960), p. $45-52$.

[15] L. A. Žyvotovskii, Theorems on the existence, uniqueness classes of solutions of functional equations with heraditary dependence (Russian), Differen. Urav. VII, 8 (1971), p. 1377- 1384. 